\title{
THE RADIAL LEMMA OF STRAUSS IN THE CONTEXT OF MORREY SPACES
}

\author{
Winfried Sickel, Dachun Yang* and Wen Yuan \\ Friedrich-Schiller-Universität Jena, Mathematisches Institut \\ Ernst-Abbe-Platz 2, D-07743 Jena, Germany; winfried.sickel@uni-jena.de \\ Beijing Normal University, School of Mathematical Sciences \\ Ministry of Education, Laboratory of Mathematics and Complex Systems \\ Beijing 100875, P. R. China; dcyang@bnu.edu.cn \\ Beijing Normal University, School of Mathematical Sciences, Ministry of Education \\ Laboratory of Mathematics and Complex Systems, Beijing 100875, P. R. China \\ and Friedrich-Schiller-Universität Jena, Mathematisches Institut \\ Ernst-Abbe-Platz 2, D-07743 Jena, Germany; wenyuan@bnu.edu.cn
}

\begin{abstract}
In this article, the authors consider smoothness and decay properties of radial functions belonging to smoothness spaces related to Morrey spaces (Sobolev-Morrey spaces, Besovtype spaces and Besov-Morrey spaces). Within this framework, some generalizations of the radial lemma of Strauss are obtained.
\end{abstract}

\section{Introduction}

At the end of the seventies, Strauss [39] was the first who observed that there exists an interplay between the regularity and the decay properties of radial functions. We recall his lemma as follows.

Radial Lemma. Let $n \geq 2$. Every radial function $f \in H^{1}\left(\mathbf{R}^{n}\right)\left(=W_{2}^{1}\left(\mathbf{R}^{n}\right)\right)$ is almost everywhere equal to a function $\tilde{f}$, continuous for $x \neq 0$, such that, for all $x \in \mathbf{R}^{n} \backslash\{0\}$,

$$
|\tilde{f}(x)| \leq C|x|^{\frac{1-n}{2}}\|f\|_{H^{1}\left(\mathbf{R}^{n}\right)},
$$

where the positive constant $C$ depends only on $n$.

Strauss stated (1.1) with the extra condition $|x| \geq 1$, but this restriction is not needed. The radial lemma contains three different assertions:

(a) the existence of a representative of $f$, which is continuous outside the origin;

doi:10.5186/aasfm.2014.3918

2010 Mathematics Subject Classification: Primary 46E35; Secondary 42C15, 42B35.

Key words: Sobolev-Morrey space, Besov-type space, Besov-Morrey space, radial function, smoothness, decay.

This work is partially supported by the 2010 Joint Research Project Between China Scholarship Council and German Academic Exchange Service (PPP) (Grant No. LiuJinOu [2010]6066). Wen Yuan is supported by the National Natural Science Foundation of China (Grant No. 11101038) and the Alexander von Humboldt Foundation. Dachun Yang is supported by the National Natural Science Foundation of China (Grant Nos. 11171027 \& 11361020). This project is also partially supported by the Specialized Research Fund for the Doctoral Program of Higher Education of China (Grant No. 20120003110003) and the Fundamental Research Funds for Central Universities of China (Grant No. 2012LYB26).

${ }^{*}$ Corresponding author. 
(b) the decay of $f$ near infinity;

(c) the limited unboundedness near the origin.

These three properties do not extend to all functions in $H^{1}\left(\mathbf{R}^{n}\right)$, of course. In particular, $H^{1}\left(\mathbf{R}^{n}\right) \not \subset L_{\infty}\left(\mathbf{R}^{n}\right), n \geq 2$. Taking an unbounded function $f \in H^{1}\left(\mathbf{R}^{n}\right)$, say, unbounded near the origin, by means of a simple shift $f\left(\cdot-x^{0}\right)$, we obtain a function which is now unbounded around $x^{0}$ and belongs to $H^{1}\left(\mathbf{R}^{n}\right)$ as well. This makes also clear that an inequality like (1.1) can not be true in the general context.

Later on, Lions [24] showed how the radial lemma extends to first order Sobolev spaces.

Proposition 1.1. Let $n \geq 2$ and $1 \leq p<\infty$. Every radial function $f \in W_{p}^{1}\left(\mathbf{R}^{n}\right)$ is almost everywhere equal to a function $\widetilde{f}$, continuous for $x \neq 0$, such that, for all $x \in \mathbf{R}^{n} \backslash\{0\}$,

$$
|\tilde{f}(x)| \leq C|x|^{\frac{1-n}{p}}\|f\|_{W_{p}^{1}\left(\mathbf{R}^{n}\right)},
$$

where the positive constant $C$ depends only on $n$ and $p$.

In recent years, after the seminal article of Kozono and Yamazaki [19], an increasing number of papers on existence and regularity of solutions of some partial differential equations is using Morrey spaces and smoothness spaces built on Morrey spaces (such as Sobolev-Morrey spaces, Besov-Morrey spaces or Besov-type spaces). For convenience of the readers, we recall their definitions (also to fix the notation which is different in different articles).

Definition 1.2. (i) Let $0<p \leq u \leq \infty$. The Morrey space $\mathcal{M}_{p}^{u}\left(\mathbf{R}^{n}\right)$ is defined to be the set of all $p$-locally Lebesgue-integrable functions $f$ on $\mathbf{R}^{n}$ such that

$$
\|f\|_{\mathcal{M}_{p}^{u}\left(\mathbf{R}^{n}\right)}:=\sup _{B}|B|^{1 / u-1 / p}\left[\int_{B}|f(x)|^{p} d x\right]^{1 / p}<\infty
$$

where the supremum is taken over all balls $B$ in $\mathbf{R}^{n}$.

(ii) Let $m \in \mathbf{N}$ and $1 \leq p \leq u \leq \infty$. The Sobolev-Morrey space $W^{m} \mathcal{M}_{p}^{u}\left(\mathbf{R}^{n}\right)$ is defined to be the collection of all functions $f \in \mathcal{M}_{p}^{u}\left(\mathbf{R}^{n}\right)$ such that all distributional derivatives $D^{\alpha} f$ of order $|\alpha| \leq m$ belong to $\mathcal{M}_{p}^{u}\left(\mathbf{R}^{n}\right)$. We equip this space with the norm

$$
\|f\|_{W^{m} \mathcal{M}_{p}^{u}\left(\mathbf{R}^{n}\right)}:=\sum_{|\alpha| \leq m}\left\|D^{\alpha} f\right\|_{\mathcal{M}_{p}^{u}\left(\mathbf{R}^{n}\right)}
$$

Remark 1.3. We call the parameter $u$ in Definition 1.2 the Morrey parameter (also in connection with the Besov-type and Besov-Morrey spaces defined below).

Obviously, we have $\mathcal{M}_{p}^{p}\left(\mathbf{R}^{n}\right)=L^{p}\left(\mathbf{R}^{n}\right)$ and $\mathcal{M}_{p}^{\infty}\left(\mathbf{R}^{n}\right)=L^{\infty}\left(\mathbf{R}^{n}\right)$. As a consequence of Hölder's inequality, we conclude the following monotonicity with respect to $p$, namely,

$$
\mathcal{M}_{w}^{u}\left(\mathbf{R}^{n}\right) \hookrightarrow \mathcal{M}_{p}^{u}\left(\mathbf{R}^{n}\right) \text { if } 0<p \leq w \leq u \leq \infty .
$$

In particular, $L^{p}\left(\mathbf{R}^{n}\right)=\mathcal{M}_{p}^{p}\left(\mathbf{R}^{n}\right) \hookrightarrow \mathcal{M}_{v}^{p}\left(\mathbf{R}^{n}\right)$ if $v<p$. This implies that

$$
W_{p}^{m}\left(\mathbf{R}^{n}\right) \hookrightarrow W^{m} \mathcal{M}_{v}^{p}\left(\mathbf{R}^{n}\right) \text { if } 1 \leq v \leq p \leq \infty .
$$

For a better understanding and later use, we mention also the embedding into the class of all complex-valued, uniformly continuous and bounded functions on $\mathbf{R}^{n}$, here 
denoted by $C_{u b}\left(\mathbf{R}^{n}\right)$. We have

$$
W^{m} \mathcal{M}_{p}^{u}\left(\mathbf{R}^{n}\right) \hookrightarrow C_{u b}\left(\mathbf{R}^{n}\right) \quad \text { if } \frac{n}{u}<m ;
$$

see [31, Proposition 17]. Our first result consists in an extension of Proposition 1.1 to Sobolev spaces built on Morrey spaces. In what follows, $C_{c}^{\infty}\left(\mathbf{R}^{n}\right)$ denotes the space of all infinitely differentiable functions on $\mathbf{R}^{n}$ with compact support.

Theorem 1.4. Let $n \geq 2$ and $1 \leq p \leq u<\infty$. Every radial function $f \in$ $W^{1} \mathcal{M}_{p}^{u}\left(\mathbf{R}^{n}\right)$ is almost everywhere equal to a function $\tilde{f}$, continuous for $x \neq 0$, such that

$$
|\widetilde{f}(x)| \leq C|x|^{\frac{1}{p}-\frac{n}{u}}\|f\|_{W^{1} \mathcal{M}_{p}^{u}\left(\mathbf{R}^{n}\right)}
$$

holds true for all $|x| \geq 1$ with some positive constant $C$ depending only on $n, p$ and $u$.

Proof. The proof is simple and (1.4) can be easily reduced to (1.2). To see this, let $f \in W^{1} \mathcal{M}_{p}^{u}\left(\mathbf{R}^{n}\right)$ be a radial function. We choose a smooth radial cut-off function

$$
\varrho \in R C_{c}^{\infty}\left(\mathbf{R}^{n}\right):=\left\{f \in C_{c}^{\infty}\left(\mathbf{R}^{n}\right): f \text { is radial }\right\}
$$

such that $0 \leq \varrho \leq 1, \operatorname{supp} \varrho \subset\left\{x \in \mathbf{R}^{n}: 1 / 2<|x|<2\right\}$, and $\sum_{j \in \mathbf{Z}} \varrho\left(2^{-j} x\right)=1$ for all $x \in \mathbf{R}^{n} \backslash\{0\}$. Then, for all $2^{k} \leq|x|<2^{k+1}$ with $k \in \mathbf{Z}$, we see that

$$
f(x)=\sum_{j \in \mathbf{Z}} \varrho\left(2^{-j} x\right) f(x)=\sum_{j=k-1}^{k+1} \rho\left(2^{-j} x\right) f(x) .
$$

Notice that, for all $k \in \mathbf{Z}_{+}:=\{0,1, \ldots\}$, the function $\sum_{j=k-1}^{k+1} \varrho\left(2^{-j} \cdot\right) f(\cdot)$ is a radial function in $W_{p}^{1}\left(\mathbf{R}^{n}\right)$ and hence, by Proposition 1.1, there exists a function $g_{k}$, which is continuous on $\mathbf{R}^{n} \backslash\{0\}$ and coincides with $\sum_{j=k-1}^{k+1} \varrho\left(2^{-j} \cdot\right) f(\cdot)$ almost everywhere, such that, for all $x \in \mathbf{R}^{n}$,

$$
\begin{aligned}
\left|g_{k}(x)\right| & \leq C|x|^{\frac{1-n}{p}} \sum_{j=k-1}^{k+1}\left\|\varrho\left(2^{-j} \cdot\right) f(\cdot)\right\|_{W_{p}^{1}\left(\mathbf{R}^{n}\right)} \\
& \leq C|x|^{\frac{1-n}{p}} \sum_{j=k-1}^{k+1} \sum_{|\alpha| \leq 1}\left[\int_{2^{k-2}<|y|<2^{k+2}}\left|D^{\alpha}\left(\varrho\left(2^{-j} \cdot\right) f\right)(y)\right|^{p} d y\right]^{1 / p} \\
& \leq C\left[\max _{|\alpha| \leq 1}\left\|D^{\alpha} \varrho\right\|_{L^{\infty}\left(\mathbf{R}^{n}\right)}\right]|x|^{\frac{1-n}{p}} \sum_{|\alpha| \leq 1}\left[\int_{2^{k-2}<|y|<2^{k+2}}\left|D^{\alpha} f(y)\right|^{p} d y\right]^{1 / p},
\end{aligned}
$$

where $C$ is a positive constant depending only on $p$ and $n$ and, in the last inequality, we used the fact that $k \in \mathbf{Z}_{+}$. Now, we switch to the Morrey norm by inserting the volume of the ball with radius $2^{k+2}$ to the power $1 / u-1 / p$. By $\omega_{n}$ we denote the volume of the unit ball. Then it follows that, for all $2^{k} \leq|x|<2^{k+1}$ with $k \in \mathbf{Z}_{+}$,

$$
\begin{aligned}
\left|g_{k}(x)\right| & \leq C\left[\max _{|\alpha| \leq 1}\left\|D^{\alpha} \varrho\right\|_{L^{\infty}\left(\mathbf{R}^{n}\right)}\right]|x|^{\frac{1-n}{p}}\left(4^{n} 2^{k n} \omega_{n}\right)^{\frac{1}{p}-\frac{1}{u}}\|f\|_{W^{1} \mathcal{M}_{p}^{u}\left(\mathbf{R}^{n}\right)} \\
& \leq C|x|^{\frac{1}{p}-\frac{n}{u}}\|f\|_{W^{1} \mathcal{M}_{p}^{u}\left(\mathbf{R}^{n}\right)},
\end{aligned}
$$

where $C$ is a positive constant depending on $p, u$ and $n$ only. 
Now let $\tilde{f}:=\sum_{k \in \mathbf{Z}} g_{k} / 3$. Since $\operatorname{supp} g_{k} \cap \operatorname{supp} g_{j}=\emptyset$ if $|j-k| \geq 5$, it follows that the summation over $k$ is finite, and hence $\tilde{f}$ is continuous on $\mathbf{R}^{n} \backslash\{0\}$ and satisfies that, for all $|x| \geq 1$,

$$
|\widetilde{f}(x)| \leq C|x|^{\frac{1}{p}-\frac{n}{u}}\|f\|_{W^{1} \mathcal{M}_{p}^{u}\left(\mathbf{R}^{n}\right)},
$$

where $C$ is a positive constant depending on $p, u$ and $n$ only. Moreover, since $g_{k}$ coincides with $\sum_{j=k-1}^{k+1} \varrho\left(2^{-j} \cdot\right) f(\cdot)$ almost everywhere, we see that

$$
\widetilde{f}(x)=\sum_{k \in \mathbf{Z}} \sum_{j=k-1}^{k+1} \varrho\left(2^{-j} x\right) f(x) / 3=\sum_{j \in \mathbf{Z}} \varrho\left(2^{-j} x\right) \sum_{k=j-1}^{j+1} f(x) / 3=f(x)
$$

for almost every $x \in \mathbf{R}^{n}$. This finishes the proof of Theorem 1.4.

Remark 1.5. (i) Obviously, by taking $u=p$, we see that the inequality (1.4) covers (1.2) when $|x| \geq 1$. Furthermore, we have the decay near infinity if $1 / p<$ $n / u$. In case $1 / p>n / u$, the inequality (1.4) can be immediately improved by (1.3) resulting in the global boundedness of all elements of $W^{1} \mathcal{M}_{p}^{u}\left(\mathbf{R}^{n}\right)$.

(ii) The elementary proof given above shows that we do not need all available information on the elements of $W^{1} \mathcal{M}_{p}^{u}\left(\mathbf{R}^{n}\right)$. Indeed, we only need that $f$ belongs to $W_{p}^{1}\left(\mathbf{R}^{n}\right)$ locally and that

$$
\sup _{B}|B|^{1 / u-1 / p}\left\{\int_{B}\left|D^{\alpha} f(x)\right|^{p} d x\right\}^{1 / p}<\infty, \quad|\alpha| \leq 1,
$$

where the supremum is taken over all balls $B$, centered in the origin and having radius larger than 1 . Such kind of spaces are usually called central Morrey spaces or local Morrey spaces. They attracted some attention in recent years; see, for example, $[1,5]$. By this point of view, it would make sense to study associated smoothness spaces. However, for the moment, we concentrate on smoothness spaces related to the original Morrey norm.

The main application of the Strauss lemma and its generalizations consists in the proof of the compactness of the embedding of the radial subspace $R W_{p}^{m}\left(\mathbf{R}^{n}\right)$ of the Sobolev space $W_{p}^{m}\left(\mathbf{R}^{n}\right)$ into Lebesgue spaces $L^{q}\left(\mathbf{R}^{n}\right)$ (see, for example, [33, 36, $17,18,35])$. Those applications are possible also for this more general situation. For this, we refer to our article [54].

In many papers, we refer, for example, to the survey [30] or the articles [33], [38], [6], [36] and [34], it has been shown that one gets a better insight into the behavior of radial functions, if one replaces the Sobolev space $W_{p}^{1}\left(\mathbf{R}^{n}\right)$ by spaces of fractional order of smoothness, for instance, Bessel potential or Besov spaces. In such a framework, $H^{1}\left(\mathbf{R}^{n}\right)$ can be replaced either by $H^{s}\left(\mathbf{R}^{n}\right)$ with $s>1 / 2$ or by $B_{2,1}^{1 / 2}\left(\mathbf{R}^{n}\right)$ to guarantee the same conclusions as in the Strauss radial lemma above. But the Bessel potential space $H^{s}\left(\mathbf{R}^{n}\right)$ with $1 / 2<s<1$ and the Besov space $B_{2,1}^{1 / 2}\left(\mathbf{R}^{n}\right)$ are much larger than $H^{1}\left(\mathbf{R}^{n}\right)$. Indeed, we have

$$
H^{1}\left(\mathbf{R}^{n}\right) \hookrightarrow H^{s}\left(\mathbf{R}^{n}\right) \hookrightarrow B_{2,1}^{1 / 2}\left(\mathbf{R}^{n}\right), \quad 1 / 2<s<1,
$$

and all the embeddings are strict. The $p$-version looks as follows

$$
W_{p}^{1}\left(\mathbf{R}^{n}\right) \hookrightarrow B_{p, 1}^{1 / p}\left(\mathbf{R}^{n}\right), \quad 1<p \leq \infty
$$


and again the embedding is strict. This motivates the use of more complicated spaces of fractional order of smoothness, which is also done here. Also, in this much more general framework, we know that

$$
W^{1} \mathcal{M}_{p}^{u}\left(\mathbf{R}^{n}\right) \hookrightarrow B_{p, 1}^{1 / p, \frac{1}{p}-\frac{1}{u}}\left(\mathbf{R}^{n}\right), \quad 1<p \leq u \leq \infty .
$$

The main aim of this article consists in proving the extension of Theorem 1.4 to the Besov-type spaces $B_{p, 1}^{1 / p, \frac{1}{p}-\frac{1}{u}}\left(\mathbf{R}^{n}\right)$ instead of the Sobolev-Morrey spaces.

This article is organized as follows. In Section 2, we recall the definition and some properties of Besov-type spaces. The main part of this section is taken by a flexibilization of the known characterizations of the spaces $B_{p, q}^{s, \tau}\left(\mathbf{R}^{n}\right)$ in terms of atoms. Section 3 is devoted to the investigation of the regularity of radial functions belonging to $B_{p, q}^{s, \tau}\left(\mathbf{R}^{n}\right)$ outside the origin. Finally, in Section 4 , we study the decay of radial functions. As a service for the reader, we reformulate the outcome for Sobolev-Morrey spaces in a separate subsection at the end of Section 4.

Finally, we make some conventions on notation. If $E$ and $F$ are two quasi-Banach spaces, then the symbol $E \hookrightarrow F$ indicates that the embedding is continuous.

Let $\mathcal{Q}$ be the collection of all dyadic cubes in $\mathbf{R}^{n}$, namely,

$$
\mathcal{Q}:=\left\{Q_{j, k}:=2^{-j}\left([0,1)^{n}+k\right): j \in \mathbf{Z}, k \in \mathbf{Z}^{n}\right\} .
$$

The symbol $\ell(Q)$ is used for the side-length of $Q$ and $j_{Q}:=-\log _{2} \ell(Q)$.

As usual, the symbol $C$ denotes a positive constant which depends only on the fixed parameters $n, s, \tau, p, q$ and probably on auxiliary functions, unless otherwise stated; its value may vary from line to line. Sometimes we use the symbol "§" instead of " $\leq$ ". The meaning of $A \lesssim B$ is given by: there exists a positive constant $C$ such that $A \leq C B$. The symbol $A \asymp B$ is used as an abbreviation of $A \lesssim B \lesssim A$.

\section{Besov-type spaces}

Sobolev and Besov spaces have been widely used in various areas of analysis such as harmonic analysis and partial differential equations. There is a rich literature with respect to these spaces, we refer, for example, to the monographs $[2,3,4,26,41,42$, 43].

Here we would like to concentrate on Besov-type spaces which are related to a relatively new family of function spaces, so-called $Q_{\alpha}$ spaces; see, for example, $[8,14,45,46]$. These spaces, in general, do not coincide with Besov-Morrey spaces (Besov spaces built on Morrey spaces), but they are not so far from each other. In the following subsection we recall definitions and state some basic properties of these scales including their relations to each other. The second subsection in this section is devoted to the study of a particular atomic decomposition of Besov-type spaces, which is adapted to the radial situation and serves as the main tool for dealing with the smoothness and decay properties of radial functions belonging to a Besov-type space.

2.1. Definitions and basic properties. Let $\mathcal{S}\left(\mathbf{R}^{n}\right)$ be the collection of all Schwartz functions on $\mathbf{R}^{n}$ endowed with the usual topology and denote by $\mathcal{S}^{\prime}\left(\mathbf{R}^{n}\right)$ its topological dual, namely, the space of all bounded linear functionals on $\mathcal{S}\left(\mathbf{R}^{n}\right)$ endowed with the weak $*$-topology. The symbol $\widehat{\varphi}$ refers to the Fourier transform of $\varphi \in \mathcal{S}^{\prime}\left(\mathbf{R}^{n}\right)$. 
We introduce the scale $B_{p, q}^{s, \tau}\left(\mathbf{R}^{n}\right)$ by using smooth dyadic decompositions of unity. Let $\varphi_{0}, \varphi \in \mathcal{S}\left(\mathbf{R}^{n}\right)$ such that

$$
\operatorname{supp} \widehat{\varphi_{0}} \subset\left\{\xi \in \mathbf{R}^{n}:|\xi| \leq 2\right\} \text { and }\left|\widehat{\varphi_{0}}(\xi)\right| \geq C \text { for }|\xi| \leq 5 / 3
$$

and

(2.2) $\operatorname{supp} \widehat{\varphi} \subset\left\{\xi \in \mathbf{R}^{n}: 1 / 2 \leq|\xi| \leq 2\right\} \quad$ and $|\widehat{\varphi}(\xi)| \geq C$ for $3 / 5 \leq|\xi| \leq 5 / 3$,

where $C$ is a positive constant independent of $\xi$.

Now we recall the notion of Besov-type spaces $B_{p, q}^{s, \tau}\left(\mathbf{R}^{n}\right)$; see [53, Definition 2.1]. In what follows, for all $j \in \mathbf{N}, x \in \mathbf{R}^{n}$ and $\varphi \in \mathcal{S}\left(\mathbf{R}^{n}\right)$, let $\varphi_{j}(x):=2^{j n} \varphi\left(2^{j} x\right)$.

Definition 2.1. Let $s \in \mathbf{R}, \tau \in[0, \infty), p, q \in(0, \infty]$ and $\varphi_{0}, \varphi \in \mathcal{S}\left(\mathbf{R}^{n}\right)$ be as in (2.1) and (2.2), respectively. The Besov-type space $B_{p, q}^{s, \tau}\left(\mathbf{R}^{n}\right)$ is defined to be the collection of all $f \in \mathcal{S}^{\prime}\left(\mathbf{R}^{n}\right)$ such that

$$
\|f\|_{B_{P}^{s, q}\left(\mathbf{R}^{n}\right)}:=\sup _{P \in \mathcal{Q}} \frac{1}{|P|^{\tau}}\left\{\sum_{j=\max \left\{j_{P}, 0\right\}}^{\infty} 2^{j s q}\left[\int_{P}\left|\varphi_{j} * f(x)\right|^{p} d x\right]^{q / p}\right\}^{1 / q}<\infty
$$

with the usual modifications made in case $p=\infty$ and/or $q=\infty$.

Remark 2.2. (i) The Besov-type space $B_{p, q}^{s, \tau}\left(\mathbf{R}^{n}\right)$ is a complete quasi-normed space, namely, a quasi-Banach space (see [53, Lemma 2.1]).

(ii) In case $\tau=0$, we are back to the standard Besov spaces, namely, $B_{p, q}^{s, 0}\left(\mathbf{R}^{n}\right)=$ $B_{p, q}^{s}\left(\mathbf{R}^{n}\right)$.

(iii) We have the monotonicity, respectively, with respect to $s$ and with respect to $q$, namely,

$$
B_{p, q_{0}}^{s_{0}, \tau}\left(\mathbf{R}^{n}\right) \hookrightarrow B_{p, q_{1}}^{s_{1}, \tau}\left(\mathbf{R}^{n}\right) \text { if } s_{0}>s_{1} \text { and } 0<q_{0}, q_{1} \leq \infty,
$$

as well as $B_{p, q_{0}}^{s, \tau}\left(\mathbf{R}^{n}\right) \hookrightarrow B_{p, q_{1}}^{s, \tau}\left(\mathbf{R}^{n}\right)$ if $q_{0} \leq q_{1}$.

(iv) Let $s \in \mathbf{R}$ and $p \in(0, \infty]$. Then, it holds true $B_{p, q}^{s, \tau}\left(\mathbf{R}^{n}\right)=B_{\infty, \infty}^{s+n(\tau-1 / p)}\left(\mathbf{R}^{n}\right)$ if either $q \in(0, \infty)$ and $\tau \in(1 / p, \infty)$, or $q=\infty$ and $\tau \in[1 / p, \infty)$; see [50]. In case $s+n(\tau-1 / p)>0$, the space $B_{\infty, \infty}^{s+n(\tau-1 / p)}\left(\mathbf{R}^{n}\right)$ is a Hölder-Zygmund space with a transparent description in terms of differences; see, for example, [41, Section 2.5.7].

(v) Of course, the spaces defined above are complicated. The definition is not really transparent. For this reason, the authors have studied in [53, Section 4.3] also their characterizations in terms of differences.

(vi) The Besov-type space $B_{p, q}^{s, \tau}\left(\mathbf{R}^{n}\right)$ and its homogeneous counterpart $\dot{B}_{p, q}^{s, \tau}\left(\mathbf{R}^{n}\right)$, restricted to the Banach space case, were first introduced by El Baraka in [9, 10, 11]. The extension to quasi-Banach spaces has been done in [47, 48]; see also [49, 22]. For a first systematic study of these spaces, we refer to the lecture note [53]; see also $[31,32,51]$.

Of particular importance for us are the following embeddings. Recall that $C_{u b}\left(\mathbf{R}^{n}\right)$ denotes the class of all complex-valued, uniformly continuous and bounded functions on $\mathbf{R}^{n}$.

Proposition 2.3. Let $s \in \mathbf{R}, \tau \in[0, \infty)$ and $p, q \in(0, \infty]$.

(i) If $s+n(\tau-1 / p)>0$, then $B_{p, q}^{s, \tau}\left(\mathbf{R}^{n}\right) \hookrightarrow C_{u b}\left(\mathbf{R}^{n}\right)$.

(ii) If $p \in(0, \infty), q \in(0, \infty], \tau \in(0,1 / p)$ and $s+n \tau-\frac{n}{p}=0$, then $B_{p, q}^{s, \tau}\left(\mathbf{R}^{n}\right) \not \subset$ $C_{u b}\left(\mathbf{R}^{n}\right)$. 
(iii) If $p \in(0, \infty)$ and $q \in(0, \infty]$, then $B_{p, q}^{0,1 / p}\left(\mathbf{R}^{n}\right) \not \subset C_{u b}\left(\mathbf{R}^{n}\right)$.

For a proof of Proposition 2.3, we refer to [53, Proposition 2.6(i)] and [31]. For the convenience of the reader, we also recall the notion of Besov-Morrey spaces as follows.

Definition 2.4. Let $\varphi_{0}$ and $\varphi \in \mathcal{S}\left(\mathbf{R}^{n}\right)$ be as in (2.1) and (2.2), respectively. Let $s \in \mathbf{R}, 0<p \leq u \leq \infty$ and $0<q \leq \infty$. Then, the Besov-Morrey space $\mathcal{N}_{u, p, q}^{s}\left(\mathbf{R}^{n}\right)$ is defined to be the set of all $f \in \mathcal{S}^{\prime}\left(\mathbf{R}^{n}\right)$ such that

$$
\|f\|_{\mathcal{N}_{u, p, q}^{s}\left(\mathbf{R}^{n}\right)}:=\left[\sum_{j=0}^{\infty} 2^{j s q}\left\|\varphi_{j} * f\right\|_{\mathcal{M}_{p}^{u}\left(\mathbf{R}^{n}\right)}^{q}\right]^{1 / q}<\infty
$$

with the usual modifications made in case $p=\infty$ and/or $q=\infty$.

Remark 2.5. (i) The Besov-Morrey spaces $\mathcal{N}_{u, p, q}^{s}\left(\mathbf{R}^{n}\right)$ represent the Besov scale built on the Morrey space $\mathcal{M}_{p}^{u}\left(\mathbf{R}^{n}\right)$. Kozono and Yamazaki [19] in 1994 and later on Mazzucato [25] have been the first who investigated spaces of this type. Indeed, they studied two, slightly different, types of spaces. The first modification consists in restricting the supremum within the definition of the Morrey norm to balls with volume $\leq 1$. Secondly, they studied homogeneous Besov-Morrey spaces $\dot{\mathcal{N}}_{u, p, q}^{s}\left(\mathbf{R}^{n}\right)$. For more information on these spaces, we refer to [19, 25, 40, 28, 52] and the surveys $[31,32,51]$.

(ii) A further family of relatives of $B_{p, q}^{s, \tau}\left(\mathbf{R}^{n}\right)$ and $\mathcal{N}_{u, p, q}^{s}\left(\mathbf{R}^{n}\right)$ has been introduced and investigated by Triebel in his recent book [44].

Lemma 2.6. Let $s \in \mathbf{R}$ and $0<p \leq u \leq \infty$.

(i) If $q \in(0, \infty)$, then $\mathcal{N}_{u, p, q}^{s}\left(\mathbf{R}^{n}\right) \hookrightarrow B_{p, q}^{s, \frac{1}{p}-\frac{1}{u}}\left(\mathbf{R}^{n}\right)$.

(ii) It holds true that $\mathcal{N}_{u, p, \infty}^{s}\left(\mathbf{R}^{n}\right)=B_{p, \infty}^{s, \frac{1}{p}-\frac{1}{u}}\left(\mathbf{R}^{n}\right)$ in the sense of equivalent quasinorms.

(iii) If $m \in \mathbf{Z}_{+}$and $1 \leq p \leq u<\infty$, then

$$
\mathcal{N}_{u, p, \min (2, p)}^{m}\left(\mathbf{R}^{n}\right) \hookrightarrow W^{m} \mathcal{M}_{p}^{u}\left(\mathbf{R}^{n}\right) \hookrightarrow \mathcal{N}_{u, p, \infty}^{m}\left(\mathbf{R}^{n}\right) .
$$

Parts (i) and (ii) of Lemma 2.6 have been proved in [29]; see also [31, Proposition 7]. There the authors argued with atomic decompositions. In addition, they have been able to show that the embedding in Lemma 2.6(i) is proper if $p<u$. Concerning Lemma 2.6(iii), we refer to Sawano [27] and [31, Lemma 5].

2.2. Radial subspaces. Let $U$ be an isometry of $\mathbf{R}^{n}$ and $g \in \mathcal{S}\left(\mathbf{R}^{n}\right)$. Then, we define $g^{U}(x):=g(U x)$ for all $x \in \mathbf{R}^{n}$. For $f \in \mathcal{S}^{\prime}\left(\mathbf{R}^{n}\right)$, we put

$$
f^{U}(g):=f\left(g^{U^{-1}}\right), \quad g \in \mathcal{S}\left(\mathbf{R}^{n}\right),
$$

where $U^{-1}$ denotes the isometry inverse to $U$. Then, $f^{U}$ is also a distribution in $\mathcal{S}^{\prime}\left(\mathbf{R}^{n}\right)$.

Let $S O\left(\mathbf{R}^{n}\right)$ be the group of rotations around the origin in $\mathbf{R}^{n}$. We say that $f \in \mathcal{S}^{\prime}\left(\mathbf{R}^{n}\right)$ is invariant with respect to $S O\left(\mathbf{R}^{n}\right)$ if $f^{U}=f$ for all $U \in S O\left(\mathbf{R}^{n}\right)$. Now we are able to define the radial subspaces which we are interested in. 
Definition 2.7. Let $s, \tau, p$ and $q$ be as in Definition 2.1. The radial subspace $R B_{p, q}^{s, \tau}\left(\mathbf{R}^{n}\right)$ of the space $B_{p, q}^{s, \tau}\left(\mathbf{R}^{n}\right)$ is defined by

$R B_{p, q}^{s, \tau}\left(\mathbf{R}^{n}\right):=\left\{f \in B_{p, q}^{s, \tau}\left(\mathbf{R}^{n}\right): f\right.$ is invariant with respect to $\left.S O\left(\mathbf{R}^{n}\right)\right\}$.

Since the linear operator $T_{U} f:=f^{U}-f$ is bounded on $B_{p, q}^{s, \tau}\left(\mathbf{R}^{n}\right)$ for all $U \in$ $S O\left(\mathbf{R}^{n}\right)$ and

$$
R B_{p, q}^{s, \tau}\left(\mathbf{R}^{n}\right)=\bigcap_{U \in S O\left(\mathbf{R}^{n}\right)} \operatorname{Ker} T_{U}
$$

we know that $R B_{p, q}^{s, \tau}\left(\mathbf{R}^{n}\right)$ is a closed subspace of $B_{p, q}^{s, \tau}\left(\mathbf{R}^{n}\right)$, and hence a quasi-Banach space with respect to the induced quasi-norm.

2.3. Atomic decompositions and regular coverings. As mentioned above, our main tool in all our investigations is the description of these radial subspaces $R B_{p, q}^{s, \tau}\left(\mathbf{R}^{n}\right)$ by means of atoms. Starting point is a rather general characterization of $B_{p, q}^{s, \tau}\left(\mathbf{R}^{n}\right)$ in terms of atoms. Since this is, in a certain sense, parallel to what has been done in Frazier and Jawerth [15, 16] and also [33], we shifted this more technical part to the Appendix at the end of this article.

Atomic decompositions are always connected with a sequence of coverings of $\mathbf{R}^{n}$. Here we are interested in a very special sequence adapted to the radial situation. To begin with, we recall some notions; see Skrzypczak in [37], but also [33, Definition 1]. The notion of an atom, we are using here, represents a certain modification of the definition given in Frazier and Jawerth [15, 16]. For any open set $Q \subset \mathbf{R}^{n}$ and $r \in(0, \infty)$, we put $r Q:=\left\{x \in \mathbf{R}^{n}: \operatorname{dist}(x, Q)<r\right\}$. Also we use the abbreviations $p^{\prime}:=p /(p-1)$ if $1<p \leq \infty$, and $p^{\prime}:=\infty$ if $0<p \leq 1$.

Definition 2.8. Let $s \in \mathbf{R}, p \in(0, \infty], r \in(0, \infty), L$ and $M$ be integers such that $L \geq 0$ and $M \geq-1$. Assume that $Q \subset \mathbf{R}^{n}$ is an open connected set with $\operatorname{diam} Q=r$.

(i) A smooth function $a$ is called a $1_{L}$-atom centered in $Q$, if $\operatorname{supp} a \subset \frac{r}{2} Q$ and

$$
\sup _{y \in \mathbf{R}^{n}}\left|\partial^{\alpha} a(y)\right| \leq 1 \quad \text { for } \quad|\alpha| \leq L .
$$

(ii) A smooth function $a$ is called an $(s, p)_{L, M}$-atom centered in $Q$, if $\operatorname{supp} a \subset \frac{r}{2} Q$,

$$
\sup _{y \in \mathbf{R}^{n}}\left|\partial^{\alpha} a(y)\right| \leq r^{s-|\alpha|-\frac{n}{p}} \quad \text { for } \quad|\alpha| \leq L,
$$

and

$$
\left|\int_{\mathbf{R}^{n}} a(y) \varphi(y) d y\right| \leq r^{s+M+1+n / p^{\prime}}\|\varphi\|_{C^{M+1}(\overline{r Q})}
$$

for all $\varphi \in C^{\infty}\left(\mathbf{R}^{n}\right)$, where $\|\varphi\|_{C^{M+1}(\overline{r Q})}:=\sup _{x \in \overline{r Q}} \sup _{|\alpha| \leq M+1}\left|\partial^{\alpha} \varphi(x)\right|$.

Remark 2.9. We recall that, when $M=-1$, then the second condition in Definition 2.8(ii) is void. Of certain use is the following simple observation: if $a$ is

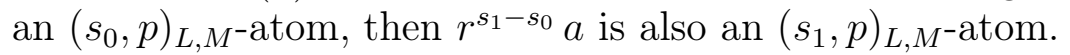

Following [33], let $\left\{Q_{\ell}\right\}_{\ell}$ be a covering of $\mathbf{R}^{n}$, where $Q_{\ell}$ for $\ell$ is a connected open set, and put

$$
C_{Q}:=\sup _{x \in \mathbf{R}^{n}} \mid\left\{\ell: Q_{\ell} \text { contains } x\right\} \mid
$$

(here $|\cdot|$ denotes the cardinality of the set), which is called the multiplicity of the covering $\left\{Q_{\ell}\right\}_{\ell}$. A covering with finite multiplicity is said to be uniformly locally 
finite. Let $\left\{\Omega_{j}\right\}_{j=0}^{\infty}:=\left\{\left\{\Omega_{j, \ell}\right\}_{\ell=0}^{\infty}\right\}_{j=0}^{\infty}$ be a sequence of uniformly locally finite coverings of $\mathbf{R}^{n}$. The supremum of multiplicities of the coverings $\left\{\Omega_{j}\right\}_{j=0}^{\infty}$ is called the multiplicity of the sequence $\left\{\Omega_{j}\right\}_{j=0}^{\infty}$.

Definition 2.10. A sequence $\left\{\Omega_{j}\right\}_{j=0}^{\infty}:=\left\{\left\{\Omega_{j, \ell}\right\}_{\ell=0}^{\infty}\right\}_{j=0}^{\infty}$ of coverings of $\mathbf{R}^{n}$ is said to be regular, if the following conditions are satisfied:

(i) $\mathbf{R}^{n} \subset \cup_{\ell \in \mathbf{Z}_{+}} \overline{\Omega_{j, \ell}}$ for all $j \in \mathbf{Z}_{+}$;

(ii) there exists some positive number $\varepsilon_{0}$ such that, for all $\varepsilon \in\left(0, \varepsilon_{0}\right)$, the sequences of coverings, $\left\{\left\{\varepsilon 2^{-j} \Omega_{j, \ell}\right\}_{\ell=0}^{\infty}\right\}_{j=0}^{\infty}$, have finite multiplicities which are uniformly bound with respect to $\varepsilon$;

(iii) there exist positive numbers $B_{n}$ and $C_{n}$, depending only on the dimension $n$, such that $\operatorname{diam} \Omega_{j, \ell} \leq B_{n} 2^{-j}$ and $C_{n} 2^{-j n} \leq\left|\Omega_{j, \ell}\right|$.

Remark 2.11. (i) By $\omega_{n}$ we denote the volume of the unit ball in $\mathbf{R}^{n}$. Let $A_{n}:=$ $\left(C_{n} / \omega_{n}\right)^{1 / n}$. Then, Definition 2.10(iii) implies that $A_{n} 2^{-j} \leq \operatorname{diam} \Omega_{j, \ell} \leq B_{n} 2^{-j}$ and, for all $j$ and $\ell, C_{n} 2^{-j n} \leq\left|\Omega_{j, \ell}\right| \leq B_{n}^{n} \omega_{n} 2^{-j n}$.

(ii) If $\left\{\left\{\Omega_{j, \ell}\right\}_{\ell=0}^{\infty}\right\}_{j=0}^{\infty}$ is a regular sequence of coverings, the cardinality of the sets,

$$
I_{j, \ell}:=\left\{k \in \mathbf{Z}_{+}: \Omega_{j, \ell} \cap \Omega_{j, k} \neq \emptyset\right\}, \quad j, \ell \in \mathbf{Z}_{+},
$$

is uniformly bounded; see [33, Lemma 1].

Next we define some sequence spaces with respect to a regular sequence $\left\{\left\{\Omega_{j, \ell}\right\}_{\ell=0}^{\infty}\right\}_{j=0}^{\infty}$ of coverings.

Definition 2.12. Let $\tau \in[0, \infty)$ and $p, q \in(0, \infty]$. Let $\Omega:=\left\{\left\{\Omega_{j, \ell}\right\}_{\ell=0}^{\infty}\right\}_{j=0}^{\infty}$ be a regular sequence of coverings. Then, the sequence space $b(p, q, \tau, \Omega)$ is defined to be the set of all sequences $t:=\left\{t_{j, \ell}\right\}_{j, \ell=0}^{\infty} \subset \mathbf{C}$ such that

$$
\|t\|_{b(p, q, \tau, \Omega)}:=\sup _{P \in \mathcal{Q}} \frac{1}{|P|^{\tau}}\left\{\sum_{j=\max \left\{j_{P}, 0\right\}}^{\infty}\left[\sum_{\substack{\ell \in \mathbf{z}_{+} \\ \Omega_{j, \ell} \neq \emptyset}}\left|t_{j, \ell}\right|^{p}\right]^{q / p}\right\}^{1 / q}<\infty .
$$

Remark 2.13. In case $\tau<1 / p$, the replacement of the sum $\sum_{j=\max \left\{j_{P}, 0\right\}}^{\infty}$ in Definition 2.12 by $\sum_{j=0}^{\infty}$ yields an equivalent quasi-norm. For a proof, we refer to [29].

Definition 2.14. Let $s \in \mathbf{R}, \tau \in[0, \infty)$ and $p, q \in(0, \infty]$. The space $B_{p, q}^{s, \tau}\left(\mathbf{R}^{n}\right)$ is said to admit an atomic decomposition with respect to the sequence $\left\{\left\{\Omega_{j, \ell}\right\}_{\ell=0}^{\infty}\right\}_{j=0}^{\infty}$, if there exist integers $L$ and $M$ such that

(i) any $f \in B_{p, q}^{s, \tau}\left(\mathbf{R}^{n}\right)$ can be represented by

$$
f=\sum_{j=0}^{\infty} \sum_{\ell=0}^{\infty} t_{j, \ell} a_{j, \ell}
$$

in $\mathcal{S}^{\prime}\left(\mathbf{R}^{n}\right)$, where $a_{0, \ell}$ is a $1_{L}$-atom centered at the set $\Omega_{0, \ell}, a_{j, \ell}$ with $j \in \mathbf{N}$ is an $(s, p)_{L, M}$-atom centered at the set $\Omega_{j, \ell}$, and $\left\{t_{j, \ell}\right\}_{j, \ell \in \mathbf{Z}_{+}}$is a sequence of complex numbers satisfying that

$$
\left\|\left\{t_{j, \ell}\right\}_{j, \ell \in \mathbf{Z}_{+}}\right\|_{b(p, q, \tau, \Omega)}<\infty
$$

(ii) any $f \in \mathcal{S}^{\prime}\left(\mathbf{R}^{n}\right)$ given by (2.3) with (2.4) is an element of $B_{p, q}^{s, \tau}\left(\mathbf{R}^{n}\right)$; 
(iii) the infimum of (2.4) with respect to all admissible representations of $f$ is an equivalent norm in $B_{p, q}^{s, \tau}\left(\mathbf{R}^{n}\right)$.

Next we show that, if $\left\{\Omega_{j}\right\}_{j=0}^{\infty}$ is a regular sequence of coverings, then, for some $L$ and $M$, the space $B_{p, q}^{s, \tau}\left(\mathbf{R}^{n}\right)$ admits an atomic decomposition with respect to $\left\{\Omega_{j}\right\}_{j=0}^{\infty}$. In what follows, the symbol $\lfloor\alpha\rfloor$ for any $\alpha \in \mathbf{R}$ denotes the maximal integer not more than $\alpha$.

Theorem 2.15. Let $s \in \mathbf{R}, \tau \in[0, \infty)$ and $p, q \in(0, \infty]$. Let $\left\{\Omega_{j}\right\}_{j=0}^{\infty}$ be a regular sequence of coverings and let $L$ and $M$ be integers such that

$$
L \geq \max \{\lfloor s+n \tau\rfloor+1,0\} \quad \text { and } \quad M \geq \max \left\{\left\lfloor\sigma_{p}-s\right\rfloor,-1\right\},
$$

where $\sigma_{p}:=n(\max \{0,1 / p-1\})$. Then, the space $B_{p, q}^{s, \tau}\left(\mathbf{R}^{n}\right)$ admits an atomic decomposition with respect to $\left\{\Omega_{j}\right\}_{j=0}^{\infty}$.

Remark 2.16. This theorem represents an extension of the well-known atomic characterization discussed by Frazier and Jawerth [15, 16]. More exactly, choosing

$$
\Omega_{j}:=\left\{Q_{j, k}: k \in \mathbf{Z}^{n}\right\}, \quad j \in \mathbf{Z}_{+},
$$

(see (1.5)) and $\tau=0$, then Theorem 2.15 has been proved in the quoted papers by Frazier and Jawerth. Regular coverings and related atomic decompositions, also restricted to $\tau=0$, have been discussed in [33]; see also [38]. Atomic decompositions of Besov-type spaces have been investigated in [53] and [23]. Our proof of Theorem 2.15 is more or less parallel to those given in the quoted papers. For that reason, we shift it into the Appendix at the end of this article.

2.4. Atomic decompositions of radial subspaces. Now, we make use of the flexibility in the choice of the regular coverings. In [33, Section 3.2], Sickel and Skrzypczak constructed a sequence of regular coverings which is well adapted to the radial situation.

A basic role in this construction is played by the following shells (balls if $k=0$ ):

$$
P_{j, k}:=\left\{x \in \mathbf{R}^{n}: k 2^{-j} \leq|x|<(k+1) 2^{-j}\right\}, \quad j, k \in \mathbf{Z}_{+} .
$$

Lemma 2.17. Let $n \geq 2$. Then, there exists a regular sequence

$$
\left\{\Omega_{j}^{R}\right\}_{j \in \mathbf{Z}_{+}}:=\left\{\left\{\Omega_{j, k, \ell}^{R}\right\}_{k \in \mathbf{Z}_{+}, \ell \in\{1, \ldots, C(n, k)\}}\right\}_{j \in \mathbf{Z}_{+}}
$$

of coverings with finite multiplicity satisfying that

(a) all $\Omega_{j, k, \ell}^{R}$ are balls with centers $y_{j, k, \ell}$ satisfying that

$$
\left|y_{j, k, \ell}\right|= \begin{cases}2^{-j}(k+1 / 2), & \text { if } k \in \mathbf{N} \\ 0, & \text { if } k=0\end{cases}
$$

(b) $P_{j, k} \subset \cup_{\ell=1}^{C(n, k)} \Omega_{j, k, \ell}^{R}$ for all $j \in \mathbf{Z}_{+}$;

(c) $\operatorname{diam} \Omega_{j, k, \ell}^{R}=12 \cdot 2^{-j}$;

(d) the sums $\sum_{k=0}^{\infty} \sum_{\ell=1}^{C(n, k)} \chi_{j, k, \ell}(x)$ are uniformly bounded in $x \in \mathbf{R}^{n}$ and $j \in \mathbf{Z}_{+}$ (here $\chi_{j, k, \ell}$ denotes the characteristic function of $\Omega_{j, k, \ell}^{R}$ );

(e) $\Omega_{j, k, \ell}^{R}=\left\{x \in \mathbf{R}^{n}: 2^{j} x \in \Omega_{0, k, \ell}^{R}\right\}, j \in \mathbf{Z}_{+}$;

(f) $C(n, 0)=1, C(n, k) \in \mathbf{N}$ and $C(n, k) \asymp k^{n-1}$, when $k \in \mathbf{N}$; 
(g) with an appropriate enumeration, it holds true that

$$
\left\{\left(x_{1}, 0, \ldots, 0\right): x_{1} \geq 0\right\} \subset \bigcup_{k=0}^{\infty} \Omega_{j, k, 1}^{R}
$$

and

$$
\left|\left\{\left(x_{1}, 0, \ldots, 0\right): x_{1} \in \mathbf{R}\right\} \cap \Omega_{j, k, 1}^{R}\right| \asymp 2^{-j} ;
$$

(h) for all $k \in \mathbf{N}$ and $\ell \in\{1, \ldots, C(n, k)\}$, there exists an element $U_{k, \ell} \in S O\left(\mathbf{R}^{n}\right)$ such that $\Omega_{j, k, \ell}^{R}=U_{k, \ell}\left(\Omega_{j, k, 1}^{R}\right)$;

(i) Let $\varepsilon \in\left(0, \varepsilon_{0}\right)$. Then, the multiplicity of the sequence

$$
\left\{\left\{\varepsilon \Omega_{j, k, \ell}^{R}: \ell \in\{1, \ldots, C(n, k)\}, k \in \mathbf{Z}_{+}\right\}\right\}_{j \in \mathbf{Z}_{+}}
$$

is finite with multiplicity constant independent of $\varepsilon$.

If $k=0$, we sometimes use the notation $\Omega_{j, 0}^{R}$ to replace $\Omega_{j, 0,1}^{R}$. Lemma 2.17(g) is not stated explicitly in [33], but it follows immediately from the construction described there; see also [20]. Most transparent is the case $n=2$. Here is a picture:

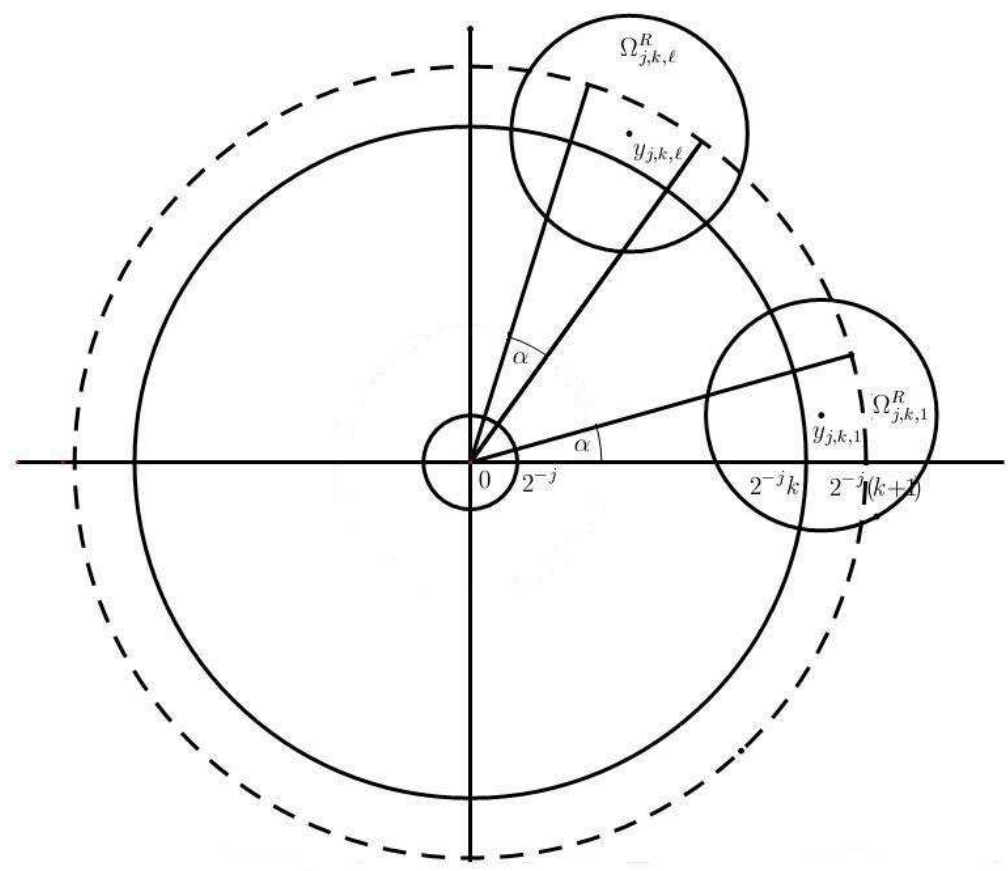

Figure 1. A piece of the covering $\left\{\Omega_{j, k, \ell}^{R}\right\}_{j=0}^{\infty}$ in case $n=2$.

Here the angle $\alpha$ is taken to be $(2 \pi) /(2 k+1), k \in \mathbf{N}$.

Applying Theorem 2.15 and Lemma 2.17, we obtain the following atomic decomposition of radial spaces. In what follows, we use the following abbreviation

$$
\omega(P, j, k):=\mid\left\{\ell \in \mathbf{N}: 1 \leq \ell \leq C(n, k) \text { and } \Omega_{j, k, \ell}^{R} \cap P \neq \emptyset\right\} \mid .
$$

Obviously, $0 \leq \omega(P, j, 0) \leq 1$ for all $j$ and dyadic cubes $P$.

Theorem 2.18. Let $n \geq 2, s \in \mathbf{R}, \tau \in[0, \infty), p, q \in(0, \infty]$, integers $L, M$ satisfy that

$$
L \geq \max \{\lfloor s+n \tau\rfloor+1,0\} \quad \text { and } \quad M \geq \max \left\{\left\lfloor\sigma_{p}-s\right\rfloor,-1\right\},
$$

and $\left\{\Omega_{j}^{R}\right\}_{j \in \mathbf{Z}_{+}}:=\left\{\left\{\Omega_{j, k, \ell}^{R}\right\}_{k \in \mathbf{Z}_{+}, \ell \in\{1, \ldots, C(n, k)\}}\right\}_{j \in \mathbf{Z}_{+}}$be as in Lemma 2.17. 
(i) Then, $R B_{p, q}^{s, \tau}\left(\mathbf{R}^{n}\right)$ admits an atomic decomposition with respect to $\left\{\Omega_{j}^{R}\right\}_{j \in \mathbf{Z}_{+}}$.

(ii) If $f \in R B_{p, q}^{s, \tau}\left(\mathbf{R}^{n}\right)$, then $f$ admits an atomic decomposition

$$
f=\sum_{j=0}^{\infty} t_{j, 0} a_{j, 0}+\sum_{j=0}^{\infty} \sum_{k=1}^{\infty} t_{j, k} \sum_{\ell=1}^{C(n, k)} a_{j, k, \ell}
$$

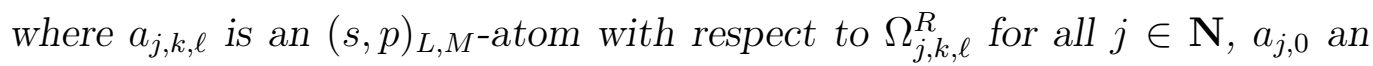
$(s, p)_{L, M}$-atom with respect to $\Omega_{j, 0}^{R}$ for all $j \in \mathbf{N}, a_{0, k, \ell}$ a $1_{L}$-atom with respect to $\Omega_{0, k, \ell}^{R}, a_{0,0}$ a $1_{L}$-atom with respect to $\Omega_{0,0}^{R}$ and

$$
\sup _{P \in \mathcal{Q}} \frac{1}{|P|^{\tau}}\left\{\sum_{j=\max \left\{j_{P}, 0\right\}}^{\infty}\left[\sum_{k=0}^{\infty} \omega(P, j, k)\left|t_{j, k}\right|^{p}\right]^{\frac{q}{p}}\right\}^{\frac{1}{q}} \leq C\|f\|_{B_{p, q}^{s, \tau}\left(\mathbf{R}^{n}\right)} .
$$

Here $C$ is a positive constant independent of $f$.

Proof. Part (i) is an immediate consequence of Theorem 2.15 and Lemma 2.17. Concerning (ii), we employ the particular decomposition constructed in Step 1 of the proof of Theorem 2.15. Rewriting (5.1), we obtain

$$
t_{j, k, \ell}:= \begin{cases}D(n, M) \sup _{y \in \Omega_{0, k, \ell}^{R}}\left|\varphi_{0} * f(y)\right|, & \text { if } j=0 ; \\ E(n, M) 2^{j(s-n / p)} \sup _{y \in \Omega_{j, k, \ell}^{R}}\left|\varphi_{j} * f(y)\right|, & \text { if } j \in \mathbf{N} .\end{cases}
$$

Since $\varphi_{0} * f$ and $\varphi_{j} * f$ are radial functions if $f, \varphi_{0}$ and $\varphi$ are radial, we conclude, by using Lemma $2.17(\mathrm{~h})$ of our regular covering, that

$$
t_{j, k, \ell}=t_{j, k, 1} \quad \text { for all } j, k, \ell \text {. }
$$

This proves (ii) and hence finishes the proof of Theorem 2.18.

Remark 2.19. Epperson and Frazier $[12,13]$ independently developed a theory of radial subspaces of Besov and Triebel-Lizorkin spaces. They preferred to work with atomic decompositions, where the atoms themselves are radial. We did not follow their treatment here, but sometimes this different point of view has some advantages.

\section{On the smoothness of radial functions outside the origin}

To begin with, we study the continuity of radial functions outside the origin. As usual, we call an element $f$ of $B_{p, q}^{s, \tau}\left(\mathbf{R}^{n}\right)$ continuous, if it has a continuous representative.

Before we start to investigate this problem, we explain how we use the atomic decomposition in (2.5). This is also of some importance for all other proofs here. The main feature is the local structure in the following sense. Let $f$ be given by (2.5), namely,

$$
f=\sum_{j=0}^{\infty} t_{j, 0} a_{j, 0}+\sum_{j=0}^{\infty} \sum_{k=1}^{\infty} t_{j, k} \sum_{\ell=1}^{C(n, k)} a_{j, k, \ell}
$$

We fix $|x| \geq 1$. Observe that, for all $j \in \mathbf{Z}_{+}$, there exists $k_{j} \in \mathbf{N}$ such that

$$
k_{j} 2^{-j} \leq|x|<\left(k_{j}+1\right) 2^{-j} \text {. }
$$


Then, employing the restrictions on the supports of our atoms, we see that $f(x)$ is given by

$$
\sum_{\left|r_{j}\right| \leq N} \sum_{\left|t_{j}\right| \leq N} \sum_{j=0}^{\infty} t_{j, k_{j}+r_{j}} a_{j, k_{j}+r_{j}, \ell_{j}+t_{j}}(x)
$$

where $N \in \mathbf{N}$ is a universal number independent of $f$ and $x$. Here $\ell_{j}$ depends on $x$. To avoid technicalities, we simply deal in our investigations with the function

$$
f^{P}(x)=\sum_{j=0}^{\infty} t_{j, k_{j}} a_{j, k_{j}, \ell_{j}}(x)
$$

instead of $f$ itself. In case $0<|x|<1$, these arguments have to be modified. Let

$$
2^{-j_{0}} \leq|x|<2^{-j_{0}+1}
$$

for some $j_{0} \in \mathbf{N}$. Then, we deal with the function

$$
f^{P}(x):=\sum_{j=0}^{j_{0}-1} t_{j, 0} a_{j, 0}(x)+\sum_{j=j_{0}}^{\infty} t_{j, k_{j}} a_{j, k_{j}, \ell_{j}}(x),
$$

as a replacement of $f$ at the point $x$. Here $k_{j}$ is defined as in (3.1).

Theorem 3.1. Let $n \geq 2, p \in(0, \infty)$ and $\tau \in[0, \infty)$. Let either $s>1 / p$ and $q \in(0, \infty]$, or $s=1 / p$ and $q \in(0,1]$. Every radial element $f$ in $B_{p, q}^{s, \tau}\left(\mathbf{R}^{n}\right)$ has a representative $\tilde{f}$ which is continuous outside of the origin.

Proof. Step 1. Let $\tau \geq 1 / p$. Then, by [53, Proposition 2.6], we know that

$$
B_{p, q}^{s, \tau}\left(\mathbf{R}^{n}\right) \hookrightarrow B_{\infty, \infty}^{s}\left(\mathbf{R}^{n}\right) \quad \text { if } s>0 .
$$

Hence, all functions in $B_{p, q}^{s, \tau}\left(\mathbf{R}^{n}\right)$ are continuous on $\mathbf{R}^{n}$, not only the radial functions.

Step 2. Let $0 \leq \tau<1 / p$. By the monotonicity of the spaces $B_{p, q}^{s, \tau}\left(\mathbf{R}^{n}\right)$ (see Remark 2.2(iii)), it suffices to deal with $B_{p, 1}^{\frac{1}{p}, \tau}\left(\mathbf{R}^{n}\right)$.

Substep 2.1. Let $|x| \geq 1$. We investigate the sequence

$$
S_{N} f^{P}(x):=\sum_{j=0}^{N} t_{j, k_{j}} a_{j, k_{j}, \ell_{j}}(x), \quad N \in \mathbf{N} .
$$

Since our atoms are at least continuous, also $S_{N} f^{P}$ is continuous. Furthermore, employing the normalization of our atoms (see Definition 2.8), we find that

$$
\left|S_{N} f^{P}(x)\right| \leq \sum_{j=0}^{N} 2^{-j(s-n / p)}\left|t_{j, k_{j}}\right|=\sum_{j=0}^{N}\left(k_{j}\right)^{\frac{1-n}{p}} 2^{-j(s-n / p)}\left(k_{j}^{n-1}\left|t_{j, k_{j}}\right|^{p}\right)^{1 / p} .
$$

Let $P$ be the smallest dyadic cube containing all points $\left(k_{j}+1\right) 2^{-j} x /|x|, j \in \mathbf{Z}_{+}$, and 0 . Hence

$$
|P| \asymp|x|^{n} \asymp\left(k_{j}+1\right)^{n} 2^{-j n} \quad \text { and } \quad \omega\left(P, j, k_{j}\right) \asymp C\left(n, k_{j}\right) \asymp k_{j}^{n-1},
$$

since $k_{j} \in \mathbf{N}$. We add a short explanation for the last relation. Take $x$ such that all components are nonnegative. Let $2^{L}<\left(k_{j}+1\right) 2^{-j} \leq 2^{L+1}$. Then, $P=Q_{-L, 0}$ (see (1.5)), and $P$ contains the part of the shell (3.1) which is contained in

$$
\left\{y=\left(y_{1}, \ldots, y_{n}\right) \in \mathbf{R}^{n}: y_{i} \geq 0 \text { for all } i\right\} .
$$


By the uniform distribution of the balls $\Omega_{j, k_{j}, \ell}^{R}$ on the shell, this implies that

$$
\left|\left\{\ell \in \mathbf{Z}_{+}: P \cap \Omega_{j, k_{j}, \ell}^{R} \neq \emptyset\right\}\right| \asymp \frac{C\left(n, k_{j}\right)}{2^{n}} .
$$

Since we ignore the dependence on $n$, the claim follows. This results in that, for all $|x| \geq 1$

$$
\begin{aligned}
\left|S_{N} f^{P}(x)\right| & \lesssim \sum_{j=0}^{N}\left(k_{j}\right)^{\frac{1-n}{p}} 2^{-j(s-n / p)}\left[\sum_{\ell=1}^{\infty} \omega(P, j, \ell)\left|t_{j, \ell}\right|^{p}\right]^{1 / p} \\
& \lesssim|x|^{n \tau} \frac{|x|^{\frac{1-n}{p}}}{|P|^{\tau}} \sum_{j=0}^{\infty} 2^{-j(s-1 / p)}\left[\sum_{\ell=1}^{\infty} \omega(P, j, \ell)\left|t_{j, \ell}\right|^{p}\right]^{1 / p} \\
& \lesssim|x|^{\frac{1-n}{p}+n \tau}\|f\|_{B_{p, 1}^{\frac{1}{p}, \tau}\left(\mathbf{R}^{n}\right)}
\end{aligned}
$$

where the last inequality follows from (2.6), and the constants behind $\lesssim$ do not depend on $f$ and $x$. Practically, the same set of inequalities as in (3.5) implies that, for all $|x| \geq 1$,

$$
\left|S_{N_{1}+N_{2}} f^{P}(x)-S_{N_{1}} f^{P}(x)\right| \lesssim \frac{|x|^{\frac{1-n}{p}+n \tau}}{|P|^{\tau}} \sum_{j=N_{1}}^{\infty} 2^{-j(s-1 / p)}\left[\sum_{\ell=1}^{\infty} \omega(P, j, \ell)\left|t_{j, \ell}\right|^{p}\right]^{1 / p} \lesssim \varepsilon,
$$

if $N_{1}$ is sufficiently large (depending on $\varepsilon$ and $|x|$ ) and $N_{2} \in \mathbf{N}$ arbitrary. Let $b>1$. Then, this implies the uniform convergence of the sequence $S_{N} f^{P}$ on any shell $\left\{x \in \mathbf{R}^{n}: 1<|x| \leq b\right\}$ to a continuous limit. Coming back to the original situation, we obtain the continuity of $f$ on these regions, which means on $|x| \geq 1$.

Substep 2.2. Let $|x|<1$. In this case, there exists a natural number $j_{0}$ such that $2^{-j_{0}} \leq|x|<2^{-j_{0}+1}$. The arguments are similar to those used in Substep 2.1 with a few modifications.

We work with

$$
S_{N} f^{P}(x):=\sum_{j=0}^{j_{0}-1} t_{j, 0} a_{j, 0}(x)+\sum_{j=j_{0}}^{N} t_{j, k_{j}} a_{j, k_{j}, \ell_{j}}(x), \quad N \geq j_{0},
$$

instead of $f$ itself. As in the previous substep, we obtain

$$
\left|S_{N} f^{P}(x)\right| \leq \sum_{j=0}^{j_{0}-1} 2^{-j(s-n / p)}\left|t_{j, 0}\right|+\sum_{j=j_{0}}^{N} 2^{-j(s-n / p)}\left|t_{j, k_{j}}\right| .
$$

To estimate the second sum on the right-hand side, we argue as in Substep 2.1. Indeed, let $P$ be the smallest dyadic cube containing $\left(k_{j}+1\right) 2^{-j} x /|x|, j \in \mathbf{Z}_{+}$, $2^{-j_{0}+1} x /|x|$ and 0 . Then,

$$
|P| \asymp|x|^{n} \asymp 2^{-j_{0} n} \quad \text { and } \quad \omega\left(P, j, k_{j}\right) \asymp C\left(n, k_{j}\right) \asymp k_{j}^{n-1}, \quad j \geq j_{0} .
$$

Thus, repeating the argument in Substep 2.1, we conclude that

$$
\sum_{j=j_{0}}^{N} 2^{-j(s-n / p)}\left|t_{j, k_{j}}\right| \lesssim|x|^{\frac{1-n}{p}+n \tau}\|f\|_{B_{p, 1}^{1 / p, \tau}\left(\mathbf{R}^{n}\right)}
$$


with a positive constant behind $\lesssim$ independent of $f, N, j_{0}$ and $x$. Observe that, for all $j \in \mathbf{Z}_{+}$,

$$
\left|t_{j, 0}\right| \lesssim 2^{-j n \tau}\|f\|_{B_{p, 1}^{1 / p, \tau}\left(\mathbf{R}^{n}\right)}
$$

see (2.6). We choose $P$ as above and use $\omega(P, j, 0)=1$. By means of this inequality, we obtain

$$
\sum_{j=0}^{j_{0}-1} 2^{-j(1 / p-n / p)}\left|t_{j, 0}\right| \lesssim\|f\|_{B_{p, 1}^{1 / p, \tau}\left(\mathbf{R}^{n}\right)} \sum_{j=0}^{j_{0}-1} 2^{-j(1 / p-n / p+n \tau)},
$$

which, together with an easy calculation, implies that

$$
\begin{aligned}
\sum_{j=0}^{j_{0}-1} 2^{-j(1 / p-n / p+n \tau)} & \lesssim \begin{cases}1, & \tau \in\left(\frac{n-1}{n p}, \frac{1}{p}\right] \\
j_{0}, & \tau=\frac{n-1}{n p} ; \\
2^{-j_{0}(1 / p-n / p+n \tau)}, & \tau \in\left[0, \frac{n-1}{n p}\right)\end{cases} \\
& \asymp \begin{cases}1, & \tau \in\left(\frac{n-1}{n p}, \frac{1}{p}\right] ; \\
1-\log _{2}|x|, & \tau=\frac{n-1}{n p} ; \\
|x|^{\frac{1-n}{p}+n \tau}, & \tau \in\left[0, \frac{n-1}{n p}\right) .\end{cases}
\end{aligned}
$$

Summarizing, we find that

$$
\left|S_{N} f^{P}(x)\right| \leq w(|x|, \tau)\|f\|_{B_{p, 1}^{1 / p, \tau}\left(\mathbf{R}^{n}\right)}
$$

where

$$
w(|x|, \tau):= \begin{cases}1, & \tau \in\left(\frac{n-1}{n p}, \frac{1}{p}\right] \\ 1-\log _{2}|x|, & \tau=\frac{n-1}{n p} \\ |x|^{\frac{1-n}{p}+n \tau}, & \tau \in\left[0, \frac{n-1}{n p}\right) .\end{cases}
$$

Having established this estimate, we may proceed as at the end of Substep 2.1 to convert it into the continuity of $f$ in all shells $(3.3), j_{0} \in \mathbf{N}$. The proof is complete.

Remark 3.2. (i) For fixed $p$ and $\tau$, the largest space $B_{p, q}^{s, \tau}\left(\mathbf{R}^{n}\right)$ with the indicated property in Theorem 3.1 is given by $B_{p, 1}^{1 / p, \tau}\left(\mathbf{R}^{n}\right)$; see Remark 2.2 and Proposition 2.3.

(ii) As mentioned in the introduction, this result has many forerunners in case $\tau=$ 0. Let us mention Strauss [39] $\left(H^{1}\left(\mathbf{R}^{n}\right)=W_{2}^{1}\left(\mathbf{R}^{n}\right)=B_{2,2}^{1}\left(\mathbf{R}^{n}\right)\right)$, Lions [24] $\left(W_{p}^{1}\left(\mathbf{R}^{n}\right)\right)$ and Sickel and Skrzypczak [33] (general case with $\tau=0$ ). A much more detailed analysis of the smoothness of radial functions belonging to some Besov or TriebelLizorkin spaces has been given in Sickel, Skrzypczak and Vybiral [36]. In particular, it is shown there that, in case $\tau=0$, the results are unimprovable within the scale $B_{p, q}^{s}\left(\mathbf{R}^{n}\right)$. More exactly, in $B_{p, q}^{1 / p}\left(\mathbf{R}^{n}\right), q>1$, there exist radial and unbounded functions $f$ such that

$$
\operatorname{supp} f \subset\left\{x \in \mathbf{R}^{n}: a<|x|<b\right\}, \quad 0<a<b<\infty .
$$

Here $a$ and $b$ are at our disposal. 


\section{Decay near infinity and controlled unboundedness near the origin of radial functions}

The proof of Theorem 3.1 allows some immediate conclusions about the pointwise behavior of radial functions. Observe that, under the restrictions of Theorem 3.1, our radial functions in $B_{p, q}^{s, \tau}\left(\mathbf{R}^{n}\right)$ are continuous outside the origin, hence pointwise inequalities make sense.

Theorem 4.1. Let $n \geq 2$ and $p \in(0, \infty)$.

(i) Let $\tau \in\left(\frac{n-1}{n p}, \infty\right), s+n(\tau-1 / p)>0$ and $q \in(0, \infty]$. Then, there exists a positive constant $C$, such that, for all $f \in R B_{p, q}^{s, \tau}\left(\mathbf{R}^{n}\right)$ and $x \in \mathbf{R}^{n}$, it holds true that

$$
|f(x)| \leq C\|f\|_{B_{p, q}^{s, \tau}\left(\mathbf{R}^{n}\right)} .
$$

(ii) Let $\tau \in\left[0, \frac{n-1}{n p}\right]$. Let either $s>1 / p$ and $q \in(0, \infty]$, or $s=1 / p$ and $q \in(0,1]$. Then, there exists a positive constant $C$ such that, for all $f \in R B_{p, q}^{s, \tau}\left(\mathbf{R}^{n}\right)$ and $|x| \geq 1$, it holds true that

$$
|f(x)| \leq C\|f\|_{B_{p, q}^{s, \tau}\left(\mathbf{R}^{n}\right)}|x|^{\frac{1-n}{p}+n \tau} .
$$

(iii) Let $\tau \in\left[0, \frac{n-1}{n p}\right]$. Let either $s>1 / p$ and $q \in(0, \infty]$, or $s=1 / p$ and $q \in(0,1]$. In addition, assume that $s \leq n\left(\frac{1}{p}-\tau\right)$. Then, there exists a positive constant $C$ such that, for all $f \in R B_{p, q}^{s, \tau}\left(\mathbf{R}^{n}\right)$ and $0<|x|<1$, it holds true that

$$
|f(x)| \leq C\|f\|_{B_{p, q}^{s, \tau}\left(\mathbf{R}^{n}\right)} \begin{cases}1-\log _{2}|x|, & \tau=\frac{n-1}{n p} \\ |x|^{\frac{1-n}{p}+n \tau}, & \tau \in\left[0, \frac{n-1}{n p}\right) .\end{cases}
$$

Proof. Step 1. For the proof of (i), namely, the continuous embedding of $B_{p, q}^{s, \tau}\left(\mathbf{R}^{n}\right)$ into a certain Hölder-Zygmund space if $s+n(\tau-1 / p)>0$, see Proposition 2.3. Of course, the restriction to radial functions is superfluous here.

Step 2. Part (ii) follows from the estimate (3.5) in combination with the embeddings for Besov-type spaces mentioned in Remark 2.2(iii).

Step 3. Part (iii) follows from the estimate (3.6), again in combination with the embeddings for Besov-type spaces mentioned in Remark 2.2(iii), which completes the proof of Theorem 4.1.

Remark 4.2. (i) Let us shortly comment on the behavior near infinity. It is an easy exercise in Fourier analysis to show that the function $f \equiv 1$ belongs to $B_{p, q}^{s, \tau}\left(\mathbf{R}^{n}\right)$ if and only if $0<p \leq \infty, \tau \geq 1 / p, s \in \mathbf{R}$ and $0<q \leq \infty$. Since this function is radial, it is immediate that functions belonging to $R B_{p, q}^{s, \tau}\left(\mathbf{R}^{n}\right)$ need not have decay near infinity. With this respect, the inequality (4.1) is optimal in case $\tau \geq 1 / p$. Next, we consider the particular case $\tau=\frac{n-1}{n p}$. Then, we have

$$
\frac{1-n}{p}+n \tau=0 \text { and } s+n(\tau-1 / p)>0 \Longleftrightarrow s>\frac{1}{p} \text {. }
$$

Hence, the passage from Theorem 4.1(i) (global boundedness and no decay) to Theorem 4.1(ii) (decay near infinity) is "continuous".

(ii) We comment on the behavior near the origin. First of all, let us mention that, in case $s>n\left(\frac{1}{p}-\tau\right)$, we have the global boundedness of all functions in $B_{p, q}^{s, \tau}\left(\mathbf{R}^{n}\right)$. Hence, the assumption $s \leq n\left(\frac{1}{p}-\tau\right)$ in Theorem 4.1(iii) is natural in our 
context. In case $\tau=0$ and $s=n / p$, one knows even more about the behavior of radial functions near the origin; see [36]. For $\tau \in\left[0, \frac{n-1}{n p}\right)$, the bound near infinity is also the bound near the origin, the decay near infinity of order $|x|^{\frac{1-n}{p}+n \tau}$ becomes controlled unboundedness of order $|x|^{\frac{1-n}{p}+n \tau}$ near the origin. Again the limiting situation $\tau=\frac{n-1}{n p}$ is of certain interest. Then, singularities of logarithmic order are possible.

(iii) For fixed $p$ and $\tau$, the largest space $B_{p, q}^{s, \tau}\left(\mathbf{R}^{n}\right)$ in (ii) and (iii) of Theorem 4.1 is given by $B_{p, 1}^{1 / p, \tau}\left(\mathbf{R}^{n}\right)$; see Remark 2.2(iii).

(iv) There exists a number of references with respect to the classical case $\tau=$ 0. Following Strauss [39] and Coleman, Glazer and Martin [7], who had proved the inequality (4.2) for radial functions belonging to $W_{2}^{1}\left(\mathbf{R}^{n}\right)$, Lions [24] gave the extension to all first order Sobolev spaces $W_{p}^{1}\left(\mathbf{R}^{n}\right)$. The extension to Besov spaces and, in particular, the existence of a bound for $s$ for the validity of these statements have been found in Sickel and Skrzypczak [33]. More detailed investigations have been undertaken in Sickel, Skrzypczak and Vybiral [36]. Let us refer also to Cho and Ozawa [6], who dealed with $H^{s}\left(\mathbf{R}^{n}\right)=B_{2,2}^{s}\left(\mathbf{R}^{n}\right), s>1 / 2$, using methods from Fourier analysis. The advantage of this approach consists in its simplicity and the fact that more information about the constant $C$ in (4.2) is given. In the framework of Morrey spaces, those investigations seem to be new.

(v) Decay properties of functions under symmetry conditions have been investigated at several places. We refer to Lions [24], Kuzin and Pohozaev [21], Skrzypczak [38] and the references given there. There also different types of symmetry constraints are investigated, for example, the block radial symmetry.

Next, we turn to the questions around sharpness in Theorem 4.1. Here we have some partial answers.

Proposition 4.3. Let $p \in(0, \infty)$ and $\tau \in[0, \infty)$.

(i) Let $p>\frac{n-1}{n}$ and $\tau \in\left[0, \frac{n-1}{n p}\right]$. Assume that either $s<1 / p$ and $q \in(0, \infty]$, or $s=1 / p$ and $q \in(1, \infty]$. Then, for all $|x| \geq 1$, there exists a sequence $\left\{f_{N}\right\}_{N \in \mathbf{N}}$ of smooth and compactly supported radial functions, depending on $x$, such that $\left\|f_{N}\right\|_{B_{p, q}^{s, \tau}\left(\mathbf{R}^{n}\right)}=1$ and $\lim _{N \rightarrow \infty}\left|f_{N}(x)\right|=\infty$.

(ii) Let $s<1 / p$ and $q \in(0, \infty]$. Then, there exist a sequence $\left\{x_{N}\right\}_{N \in \mathbf{N}}$ of points in $\mathbf{R}^{n}$ and a corresponding sequence $\left\{f_{N}\right\}_{N \in \mathbf{N}}$ of smooth and compactly supported radial functions such that $x_{N} \rightarrow 0$ as $N \rightarrow \infty,\left\|f_{N}\right\|_{B_{p, q}^{s, \tau}\left(\mathbf{R}^{n}\right)} \leq 1$ and $\left|f_{N}\left(x_{N}\right)\right| \geq N\left|x_{N}\right|^{\frac{1-n}{p}+n \tau}$ for all $N \in \mathbf{N}$.

Proof. Step 1. In this step, we show (i). We follow ideas used in [33, pp. 651-653]. Let $\psi \in C_{c}^{\infty}\left(\mathbf{R}^{n}\right)$ such that $0 \leq \psi \leq 1, \psi(x)=1$ if $|x| \leq 12$ and $\psi(x)=0$ if $|x| \geq 13$. Denote by $y_{j, k, \ell}$ the center of the ball $\Omega_{j, k, \ell}^{R}$ and let

$$
\widetilde{\psi}_{j, 0}(\cdot):=\psi\left(2^{j} \cdot\right) \quad \text { and } \quad \widetilde{\psi}_{j, k, \ell}(\cdot):=\psi\left(2^{j}\left(\cdot-y_{j, k, \ell}\right)\right)
$$

for all $j \in \mathbf{Z}_{+}, k \in \mathbf{N}$ and $\ell \in\{1, \ldots, C(n, k)\}$. Then, by Lemma 2.17, we see that

$$
1 \leq \widetilde{\psi}_{j, 0}+\sum_{k \in \mathbf{N}} \sum_{\ell=1}^{C(n, k)} \widetilde{\psi}_{j, k, \ell} \leq M m_{0}
$$


where $m_{0}$ denotes the multiplicity of the sequence of coverings, $\left\{\Omega_{j, k, \ell}^{R}\right\}_{\ell}$, and $M$ is a positive constant independent of $j$. Define

and

$$
\psi_{j, 0}:=\frac{\widetilde{\psi}_{j, 0}}{\widetilde{\psi}_{j, 0}+\sum_{m \in \mathbf{N}} \sum_{r=1}^{C(n, m)} \widetilde{\psi}_{j, m, r}}
$$

Then,

$$
\psi_{j, k, \ell}:=\frac{\widetilde{\psi}_{j, k, \ell}}{\widetilde{\psi}_{j, 0}+\sum_{m \in \mathbf{N}} \sum_{r=1}^{C(n, m)} \widetilde{\psi}_{j, m, r}} .
$$

$$
\psi_{j, 0}+\sum_{m \in \mathbf{N}} \sum_{r=1}^{C(n, m)} \psi_{j, m, r} \equiv 1 .
$$

Let $\psi_{0} \in C^{\infty}([0, \infty))$ be such that $0 \leq \psi_{0} \leq 1, \psi_{0}(x)=1$ when $0 \leq x \leq 1$, and $\psi_{0}(x)=0$ when $x \geq 2$. Define

$$
\varphi_{j, r}(x):=\psi_{0}\left(\left|2^{j}\right| x\left|-2^{r}\right|\right), \quad x \in \mathbf{R}^{n},
$$

for all $j, r \in \mathbf{Z}$. Obviously, these functions are radial,

$$
\operatorname{supp} \varphi_{j, r} \subset\left\{x \in \mathbf{R}^{n}: \max \left\{0,2^{-j}\left(2^{r}-2\right)\right\} \leq|x| \leq 2^{-j}\left(2+2^{r}\right)\right\},
$$

and

$$
\varphi_{j, r}(x)=1 \quad \text { if } \quad|x|=2^{r-j} .
$$

For this family of functions, it has been proved in [33, p. 652] that

$$
\varphi_{j, r}=\sum_{k=2^{r}-r_{0}}^{2^{r}+r_{0}} \sum_{\ell=1}^{C(n, k)} \psi_{j, k, \ell} \varphi_{j, r} \quad \text { if } r \geq \max \{j+4,5\},
$$

where $r_{0}$ is independent of $j$ and $r$. Assume that $n\left(\max \left\{0, \frac{1}{p}-1\right\}\right)<s<1 / p$. In this region, no moment conditions are needed for our atoms. For some positive constant

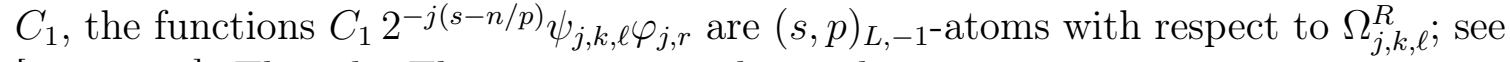
[33, p. 652]. Then, by Theorem 2.18 , we know that

$$
\begin{aligned}
\left\|\varphi_{j, r}\right\|_{B_{p, q}^{s, \tau}\left(\mathbf{R}^{n}\right)} & =\left\|\sum_{k=2^{r}-r_{0}}^{2^{r}+r_{0}} \sum_{\ell=1}^{C(n, k)} \psi_{j, k, \ell} \varphi_{j, r}\right\|_{B_{p, q}^{s, \tau}\left(\mathbf{R}^{n}\right)} \\
& \asymp \sup _{\substack{P \in \mathcal{Q} \\
j_{P} \leq j}} \frac{1}{|P|^{\tau}}\left[\sum_{k=2^{r}-r_{0}}^{2^{r}+r_{0}} \omega(P, j, k) 2^{j(s-n / p) p}\right]^{1 / p} ;
\end{aligned}
$$

see (2.6). From our knowledge about the support of $\varphi_{j, r}$ (see (4.4)), it becomes clear that the supremum is realized by dyadic cubes with side-length $\leq C\left(2^{r}+r_{0}\right) 2^{-j}$ for some positive constant $C$ independent of $r$ and $j$. Hence, the supremum in the previous formula runs only over those dyadic cubes $P$ such that

$$
2^{-j} \leq \ell(P) \lesssim\left(2^{r}+r_{0}\right) 2^{-j} \lesssim 2^{r-j}
$$

If $j_{P}=j-m$ for some $m \in \mathbf{Z}_{+}$, then, for those cubes, $m$ satisfies $0 \lesssim m \lesssim r$. Finally, the number of balls $\Omega_{j, k, \ell}^{R}$ satisfying $\Omega_{j, k, \ell}^{R} \cap P \neq \emptyset$ is at most a constant multiple of $2^{m(n-1)}$, namely,

$$
\omega(P, j, k) \lesssim 2^{m(n-1)}
$$


To prove this claim, we proceed as follows. Let $12 P_{j, k}$ denote the the annulus

$$
\left\{x \in \mathbf{R}^{n}:\left(k-\frac{11}{2}\right) 2^{-j} \leq|x|<\left(k+\frac{13}{2}\right) 2^{-j}\right\} \quad \text { if } k>\frac{11}{2},
$$

or the ball $B\left(0,\left(k+\frac{13}{2}\right) 2^{-j}\right)$ if $k \leq \frac{11}{2}$. From $\operatorname{diam} \Omega_{j, k, \ell}^{R}=12 \cdot 2^{-j}$ and the position of the centers $y_{j, k, \ell}$ of the balls $\Omega_{j, k, \ell}^{R}$ in Lemma 2.17(a), we deduce that

$$
\bigcup_{\ell=1}^{C(n, k)} \Omega_{j, k, \ell}^{R} \subset 12 P_{j, k} .
$$

On the other hand, notice that the volume of $P \cap\left(12 P_{j, k}\right)$ is at most a constant multiple of $2^{-j} \times 2^{-j_{P}(n-1)}=2^{-j n} 2^{m(n-1)}$, which can be attained in case diam $\left(P \cap 12 P_{j, k}\right) \asymp$ $\sqrt{n} \ell(P)$. Since $\left|\Omega_{j, k, \ell}^{R}\right| \asymp 2^{-j n}$ and $\left\{\Omega_{j, k, \ell}^{R}\right\}_{\ell \in\{1, \ldots, C(n, k)\}}$ is of finite multiplicity, there exists at most a positive constant multiple of $2^{m(n-1)}$ balls in $\left\{\Omega_{j, k, \ell}^{R}\right\}_{\ell \in\{1, \ldots, C(n, k)\}}$ such that

$$
\Omega_{j, k, \ell}^{R} \cap P=\Omega_{j, k, \ell}^{R} \cap\left(P \cap 12 P_{j, k}\right) \neq \emptyset .
$$

Thus, (4.7) holds true. In the picture below, we draw a cube $P, \ell(P)=2^{-j_{P}}>2^{-j}$ in a position, where $\omega(P, j, k)$ becomes maximal within the family of dyadic cubes $Q$ of side-length $2^{-j_{P}}$.

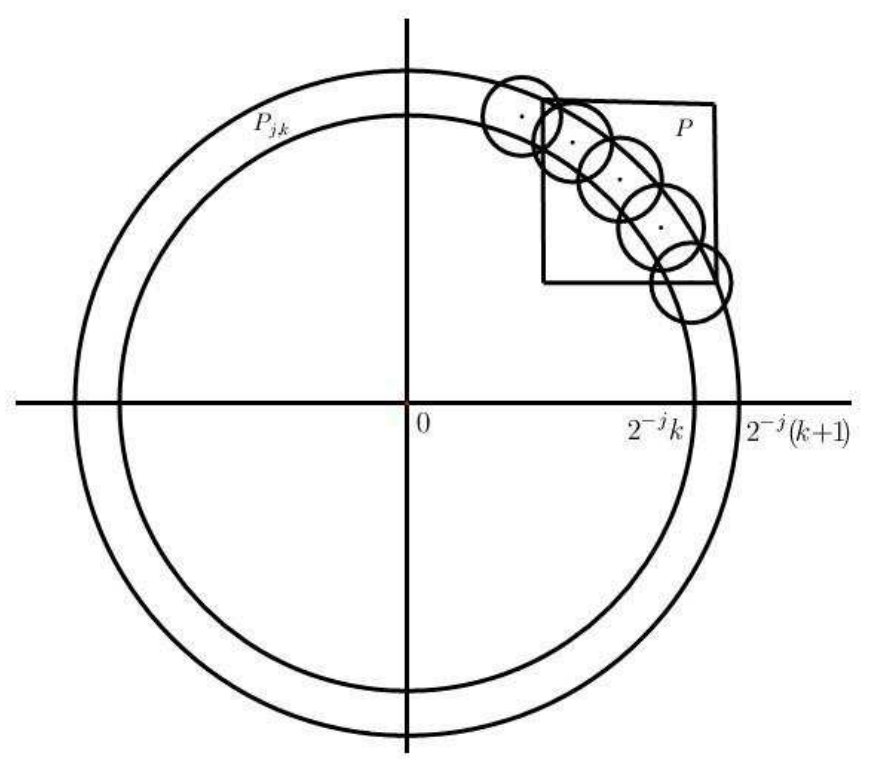

Figure 2. A dyadic cube $P$ in a position with $\omega(P, j, k)$ large.

Therefore, employing $\tau \leq(n-1) /(n p)$, (4.6) and (4.7), we obtain

$$
\left\|\varphi_{j, r}\right\|_{B_{p, q}^{s, \tau}\left(\mathbf{R}^{n}\right)} \lesssim \sup _{0 \leq m \lesssim r} 2^{(j-m) n \tau} 2^{j(s-n / p)} 2^{m(n-1) / p} \asymp 2^{j(s-n / p+n \tau)} 2^{r\left(\frac{n-1}{p}-n \tau\right)} .
$$

Now we turn to a new normalization

$$
\left\|2^{-j(s-n / p+n \tau)} 2^{-r\left(\frac{n-1}{p}-n \tau\right)} \varphi_{j, r}\right\|_{B_{p, q}^{s, \tau}\left(\mathbf{R}^{n}\right)} \leq 1,
$$


but, thanks to (4.5), we find that

$$
\begin{aligned}
2^{-j(s-n / p+n \tau)} 2^{-r\left(\frac{n-1}{p}-n \tau\right)} \varphi_{j, r}\left(\left(2^{r-j}, 0, \ldots, 0\right)\right) & =2^{-j(s-n / p+n \tau)} 2^{-r\left(\frac{n-1}{p}-n \tau\right)} \\
& =2^{j(1 / p-s)} 2^{(r-j)\left(-\frac{n-1}{p}+n \tau\right)}
\end{aligned}
$$

Let $|x|:=2^{t}$ for some $t \geq 5$. We apply the preceding formulas with $t:=r-j$ fixed and $j$ tending to infinity. This proves the desired result in the case of dyadic values of $|x|$. The general case $|x| \geq 1$ follows from using the same arguments with some minor modifications. Moreover, by the monotonicity of the quasi-norms with respect to $s$ and $q$, we also obtain the desired result in case $s \leq n(\max \{0,1 / p-1\})$.

Now, we consider the case that $s=1 / p$ and $q \in(1, \infty]$. By the assumption on $p$, we know that $s>n(\max \{0,1 / p-1\})$, which means that the atoms in Theorem 2.18 do not need to satisfy any moment condition. Let

$$
\varphi_{N, r}=\sum_{j=1}^{N} \alpha_{j} \varphi_{j, j+4+r}, \quad N \in \mathbf{N}, \quad r \geq 0
$$

where $\left\{\alpha_{j}\right\}_{j}$ is a sequence of positive numbers such that $\left\{\alpha_{j}\right\}_{j} \in \ell^{q}$ but $\left\{\alpha_{j}\right\}_{j} \notin \ell^{1}$. Then, by an argument similar to that used in case $s<1 / p$, we see that

$$
\left\|\varphi_{N, r}\right\|_{B_{p, q}^{s, \tau}\left(\mathbf{R}^{n}\right)} \asymp \sup _{P \in \mathcal{Q}} \frac{1}{|P|^{\tau}}\left\{\sum_{j=\max \left(j_{P}, 1\right)}^{N} \alpha_{j}^{q}\left[\sum_{k=2^{j+4+r}-r_{0}}^{2^{j+4+r}+r_{0}} \omega(P, j, k) 2^{j(1-n)}\right]^{q / p}\right\}^{1 / q} .
$$

Arguing as above, in the supremum, only those dyadic cubes $P$ such that $2^{-N} \leq$ $\ell(P) \lesssim 2^{r}$ are of relevance; see (4.4). Thus, $-r \lesssim j_{P} \leq N$. By the same argument as that used in (4.7), we see that, for all $j \geq j_{P}, \omega(P, j, k) \lesssim 2^{\left(j-j_{P}\right)(n-1)}$. Therefore, it follows, from $\tau \in\left[0, \frac{n-1}{n p}\right]$, that

$$
\left\|\varphi_{N, r}\right\|_{B_{P, q}^{s, \tau}\left(\mathbf{R}^{n}\right)} \lesssim \sup _{\substack{P \in \mathcal{Q} \\-r \lesssim j_{P} \leq N}} 2^{j_{P}[n \tau-(n-1) / p]}\left\{\sum_{j=\max \left(j_{P}, 1\right)}^{N} \alpha_{j}^{q}\right\}^{1 / q} \lesssim 2^{r\left(\frac{n-1}{p}-n \tau\right)}\left\{\sum_{j=1}^{N} \alpha_{j}^{q}\right\}^{1 / q} .
$$

Next, observe that

$$
\varphi_{N, r}\left(\left(2^{r+4}, 0, \ldots, 0\right)\right)=\sum_{j=1}^{N} \alpha_{j} \rightarrow \infty \quad \text { as } N \rightarrow \infty
$$

see (4.5). By taking $r$ fixed, but letting $N$ tend to infinity, we then complete the proof for the case of dyadic values of $|x|$. The general case $|x| \geq 1$ follows from some minor modifications.

Step 2. In this step, we show (ii). Assume first that $n(\max \{0,1 / p-1\})<s<$

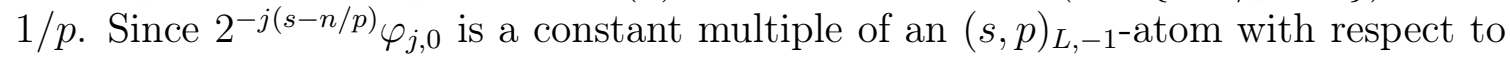
$\Omega_{j, 0}^{R}$, by Theorem 2.18 , we know that

$$
\left\|2^{-j n \tau} 2^{-j(s-n / p)} \varphi_{j, 0}\right\|_{B_{p, q}^{s, \tau}\left(\mathbf{R}^{n}\right)} \lesssim 1 .
$$

Considering the function $2^{-j n \tau} 2^{-j(s-n / p)} \varphi_{j, 0}$ on the ring $|x|=2^{-j}$, we know that

$$
2^{-j n \tau} 2^{-j(s-n / p)}\left|\varphi_{j, 0}(x)\right|=|x|^{s-n / p+n \tau} \geq N|x|^{\frac{1-n}{p}+n \tau}
$$


when $|x|$ is small enough. The general case $s<1 / p$ follows from a monotonicity argument. This finishes the proof of Proposition 4.3.

Remark 4.4. (i) Combining Proposition 4.3 and Theorem 4.1(ii), we know that the inequality (4.2) can not be true if either $s<1 / p$ and $q$ arbitrary, or $s=1 / p$ and $1<q \leq \infty$.

(ii) Proposition 4.3(ii) in combination with Theorem 4.1(iii) implies that, in case $s<1 / p$, the inequality (4.3) can not be true.

(iii) In case $\tau=0$, a much more detailed investigation of the sharpness of the results has been undertaken in [36]. However, the approach used there is based on a characterization of the trace spaces of $R B_{p, q}^{s}\left(\mathbf{R}^{n}\right)$. That would be of certain interest also in the present situation.

Finally, for the convenience of the reader, we formulate the consequences of Theorems 3.1 and 4.1, and Proposition 4.3 in case of Sobolev-Morrey spaces. Applying the embedding

$$
W^{m} \mathcal{M}_{p}^{u}\left(\mathbf{R}^{n}\right) \hookrightarrow \mathcal{N}_{u, p, \infty}^{m}\left(\mathbf{R}^{n}\right)=B_{p, \infty}^{m, \tau}\left(\mathbf{R}^{n}\right), \quad \tau=\frac{1}{p}-\frac{1}{u},
$$

and Theorem 3.1, we have the following conclusion.

Corollary 4.5. Let $n \geq 2,1<p \leq u<\infty$ and $m \in \mathbf{N}$. Then, every radial element $f$ in $W^{m} \mathcal{M}_{p}^{u}\left(\mathbf{R}^{n}\right)$ has a representative $\tilde{f}$ which is continuous outside of the origin.

The next result follows from (4.8) and Theorem 4.1.

Corollary 4.6. Let $n \geq 2,1<p \leq u<\infty$ and $m \in \mathbf{N}$.

(i) Let $u / p \leq n$. Then, there exists a positive constant $C$ such that, for all $f \in R W^{m} \mathcal{M}_{p}^{u}\left(\mathbf{R}^{n}\right)$ and $|x| \geq 1$, it holds true that

$$
|f(x)| \leq C\|f\|_{W^{m} \mathcal{M}_{p}^{u}\left(\mathbf{R}^{n}\right)}|x|^{\frac{1}{p}-\frac{n}{u}} .
$$

(ii) Let $u / p \leq n$. Then, there exists a positive constant $C$ such that, for all $f \in R W^{m} \mathcal{M}_{p}^{u}\left(\mathbf{R}^{n}\right)$ and $0<|x|<1$, it holds true that

$$
|f(x)| \leq C\|f\|_{W^{m} \mathcal{M}_{p}^{u}\left(\mathbf{R}^{n}\right)} \begin{cases}1-\log _{2}|x|, & \frac{1}{p}=\frac{n}{u} \\ |x|^{\frac{1}{p}-\frac{n}{u}}, & \frac{1}{p}<\frac{n}{u} .\end{cases}
$$

Remark 4.7. Let us mention that, because of the embedding (1.3), only those situations are of interest where $n \geq u$ holds true. Of course, Corollary 4.6(i) represents Theorem 1.4 proved in a totally different way.

\section{Appendix-Proof of Theorem 2.15}

Step 1. Recall that $B_{p, q}^{s, \tau}\left(\mathbf{R}^{n}\right)=B_{\infty, \infty}^{s+n(\tau-1 / p)}\left(\mathbf{R}^{n}\right)$ if either $q \in(0, \infty)$ and $\tau \in$ $(1 / p, \infty)$, or $q=\infty$ and $\tau \in[1 / p, \infty)$ (see [50]). The desired conclusion in the above case and in case $\tau=0$ has been obtained in [33, Proposition 1].

Step 2. Next, we assume that either $\tau \in(0,1 / p)$ and $q \in(0, \infty]$, or $\tau=1 / p$ and $q \in(0, \infty)$. 
Substep 2.1. Let $f \in B_{p, q}^{s, \tau}\left(\mathbf{R}^{n}\right)$ be given. We show the existence of an appropriate atomic decomposition. Let $\psi_{0}, \psi \in \mathcal{S}\left(\mathbf{R}^{n}\right)$ be such that

$$
\left|\widehat{\psi_{0}}(\xi)\right|>0 \text { when }|\xi| \leq 2 \text {, and }|\widehat{\psi}(\xi)|>0 \text { when } 1 / 2 \leq|\xi| \leq 2 \text {. }
$$

Then, there exist $\varphi_{0}, \varphi \in \mathcal{S}\left(\mathbf{R}^{n}\right)$ such that

$$
\operatorname{supp} \widehat{\varphi_{0}} \subset\left\{\xi \in \mathbf{R}^{n}:|\xi| \leq 2\right\} \text { and }\left|\widehat{\varphi_{0}}(\xi)\right|>0 \text { when }|\xi| \leq 1,
$$

$\varphi$ satisfies $(2.2)$, and

$$
\widehat{\psi_{0}}(\xi) \overline{\widehat{\varphi_{0}}(\xi)}+\sum_{j \in \mathbf{N}} \widehat{\psi}\left(2^{-j} \xi\right) \overline{\widehat{\varphi}\left(2^{-j} \xi\right)}=1
$$

for all $\xi \in \mathbf{R}^{n}$; see Frazier and Jawerth $[15,16]$. Let $A_{n}$ be the same as in Remark 2.11(i). In addition, we may assume that

$$
\operatorname{supp} \psi_{0}, \operatorname{supp} \psi \subset\left\{x \in \mathbf{R}^{n}:|x| \leq A_{n} / 2\right\}
$$

and

$$
\int_{\mathbf{R}^{n}} x^{\gamma} \psi(x) d x=0 \quad \text { for all }|\gamma| \leq M
$$

see [33, Proposition 1]. Then, by the Calderón reproducing formula, we see that

$$
f(x)=\int_{\mathbf{R}^{n}} \psi_{0}(x-y) \widetilde{\varphi}_{0} * f(y) d y+\sum_{j \in \mathbf{N}} \int_{\mathbf{R}^{n}} \psi_{j}(x-y) \widetilde{\varphi}_{j} * f(y) d y
$$

in $\mathcal{S}^{\prime}\left(\mathbf{R}^{n}\right)$, where $\widetilde{\varphi}_{0}(\cdot)=\overline{\varphi_{0}(-\cdot)}$ and $\widetilde{\varphi}_{j}(\cdot)=\overline{\varphi_{j}(-\cdot)}$. For all $j, \ell \in \mathbf{Z}_{+}$, we define

$$
t_{j, \ell}:= \begin{cases}D(n, M) \sup _{y \in \Omega_{0, \ell}}\left|\widetilde{\varphi}_{0} * f(y)\right|, & j=0 \\ E(n, M) 2^{j(s-n / p)} \sup _{y \in \Omega_{j, \ell}}\left|\widetilde{\varphi}_{j} * f(y)\right|, & j \in \mathbf{N},\end{cases}
$$

where

$$
D(n, M):=B_{n}^{n} w_{n}\left[\max _{|\alpha| \leq L}\left\|D^{\alpha} \Psi\right\|_{C\left(\mathbf{R}^{n}\right)}\right]
$$

and

$$
\begin{aligned}
E(n, M):= & B_{n}^{n} w_{n}\left[\max _{|\alpha| \leq L}\left\|D^{\alpha} \psi\right\|_{C\left(\mathbf{R}^{n}\right)}\right] \max _{|\alpha| \leq L}\left\{A_{n}^{-s+n / p+|\alpha|}, B_{n}^{-s+n / p+|\alpha|}\right\} \\
& \times \max \left\{1, w_{n}(3 / 2)^{M+n+1} \sum_{|\gamma|=M+1} \frac{1}{\gamma !}\right\} .
\end{aligned}
$$

As in [33, Proposition 1], for all $j \in \mathbf{Z}_{+}$, we let $\Omega_{j, 0}^{*}:=\Omega_{j, 0}$ and

$$
\Omega_{j, \ell}^{*}:=\Omega_{j, \ell} \backslash\left(\bigcup_{m=0}^{\ell-1} \Omega_{j, m}\right) \text { for } \ell \in \mathbf{N} .
$$


Then, $\left\{\Omega_{j, \ell}^{*}\right\}_{\ell \in \mathbf{Z}_{+}}$is a family of pairwise disjoint sets and satisfies that $\cup_{\ell \in \mathbf{Z}_{+}} \Omega_{j, \ell}^{*}=\mathbf{R}^{n}$ for all $j \in \mathbf{Z}_{+}$. When $t_{j, \ell} \neq 0$, we let

$$
a_{j, \ell}(x):= \begin{cases}\frac{1}{t_{0, \ell}} \int_{\Omega_{0, \ell}^{*}} \psi_{0}(x-y) \widetilde{\varphi}_{0} * f(y) d y, & j=0 ; \\ \frac{1}{t_{j, \ell}} \int_{\Omega_{j, \ell}^{*}} \psi_{j}(x-y) \widetilde{\varphi}_{j} * f(y) d y, & j \in \mathbf{N} .\end{cases}
$$

When $t_{j, \ell}=0$, we define $a_{j, \ell}(x) \equiv 0$. Then, by the argument used in the proof of [33, Proposition 1], we know that $a_{0, \ell}$ is a $1_{L^{-}}$atom centered at the set $\Omega_{0, \ell}, a_{j, \ell}$ with $j \in \mathbf{N}$ an $(s, p)_{L, M}$-atom centered at the set $\Omega_{j, \ell}$ and $f=\sum_{j=0}^{\infty} \sum_{\ell=0}^{\infty} t_{j, \ell} a_{j, \ell}$ in $\mathcal{S}^{\prime}\left(\mathbf{R}^{n}\right)$. Now, we prove that

$$
\left\|\left\{t_{j, \ell}\right\}_{j, \ell \in \mathbf{Z}_{+}}\right\|_{b(p, q, \tau, \Omega)} \lesssim\|f\|_{B_{p, q}^{s, \tau}\left(\mathbf{R}^{n}\right)} .
$$

By definition of the $t_{j, \ell}$, we find that

$$
\begin{aligned}
& \left\|\left\{t_{j, \ell}\right\}_{j, \ell \in \mathbf{Z}_{+}}\right\|_{b(p, q, \tau, \Omega)} \\
& \asymp \sup _{P \in \mathcal{Q}} \frac{1}{|P|^{\tau}}\left\{\sum_{j=\max \left\{j_{P}, 0\right\}}^{\infty} 2^{j(s-n / p) q}\left[\sum_{\substack{\ell \in \mathbf{Z}_{+} \neq \emptyset \in \Omega_{j, \ell} \\
\Omega_{j, \ell} \cap P \neq \emptyset}} \sup _{y=\left.\widetilde{\varphi}_{j} * f(y)\right|^{p}}\right]^{q / p}\right\}^{1 / q} .
\end{aligned}
$$

Via an argument similar to that used in the proof of $[15,(2.11)]$, we conclude that

$$
\sup _{y \in \Omega_{j, \ell}}\left|\widetilde{\varphi}_{j} * f(y)\right|^{p} \lesssim 2^{j n} \sum_{k \in \mathbf{Z}^{n}}(1+|k|)^{-N} \int_{\Omega_{j, \ell}}\left|\widetilde{\varphi}_{j} * f\left(y+2^{-j} k\right)\right|^{p} d y,
$$

where $N$ is at our disposal. On the other hand, since diam $\Omega_{j, \ell} \leq B_{n} 2^{-j}$, there exists a constant $d \in[1, \infty)$ such that, for all $j \geq j_{P}$,

$$
\bigcup_{\ell \in \mathbf{Z}_{+}, \Omega_{j, \ell} \cap P \neq \emptyset} \Omega_{j, \ell} \subset d P .
$$

Denote by $y_{P}$ the center of $P$ and by $B(y, R)$ the ball with radius $R$ and center in $y$. We then see that

$$
\begin{aligned}
& \left\|\left\{t_{j, \ell}\right\}_{j, \ell \in \mathbf{Z}_{+}}\right\|_{b(p, q, \tau, \Omega)} \\
& \lesssim \sup _{P \in \mathcal{Q}} \frac{1}{|P|^{\tau}}\left\{\sum_{j=\max \left\{j_{P}, 0\right\}}^{\infty} 2^{j s q}\left[\sum_{\substack{\ell \in \mathbf{Z}_{+} \\
\Omega_{j, \ell} \cap \neq \emptyset}} \sum_{k \in \mathbf{Z}^{n}} \frac{\int_{\Omega_{j, \ell}}\left|\widetilde{\varphi}_{j} * f\left(y+2^{-j} k\right)\right|^{p} d y}{(1+|k|)^{N}}\right]^{q / p}\right\}^{1 / q} \\
& \lesssim \sup _{P \in \mathcal{Q}} \frac{1}{|P|^{\tau}}\left\{\sum_{j=\max \left\{j_{P}, 0\right\}}^{\infty} 2^{j s q}\left[\sum_{k \in \mathbf{Z}^{n}} \frac{\int_{d P}\left|\widetilde{\varphi}_{j} * f\left(y+2^{-j} k\right)\right|^{p} d y}{(1+|k|)^{N}}\right]^{q / p}\right\}^{1 / q} \\
& \lesssim \sup _{P \in \mathcal{Q}} \frac{1}{|P|^{\tau}}\left\{\sum_{j=\max \left\{j_{P}, 0\right\}}^{\infty} 2^{j s q}\left[\sum_{k \in \mathbf{Z}^{n}} \frac{\int_{B\left(y_{P}, d \ell(P)+|k| \ell(P)\right)}\left|\widetilde{\varphi}_{j} * f(z)\right|^{p} d z}{(1+|k|)^{N}}\right]^{q / p}\right\}^{1 / q} .
\end{aligned}
$$


Temporarily we assume $q \leq p$. By the definition of $\|f\|_{B_{p, q}^{s, \tau}\left(\mathbf{R}^{n}\right)}$, we conclude that

$$
\begin{aligned}
& \left\|\left\{t_{j, \ell}\right\}_{j, \ell \in \mathbf{Z}_{+}}\right\|_{b(p, q, \tau, \Omega)} \\
& \lesssim \sup _{P \in \mathcal{Q}} \frac{1}{|P|^{\tau}}\left\{\sum_{k \in \mathbf{Z}^{n}}(1+|k|)^{-N q / p} \sum_{j=\max \left\{j_{P}, 0\right\}}^{\infty} 2^{j s q}\left[\int_{B\left(y_{P}, d \ell(P)+|k| \ell(P)\right)}\left|\widetilde{\varphi}_{j} * f(z)\right|^{p} d z\right]^{q / p}\right\}^{1 / q} \\
& \lesssim\|f\|_{B_{p, q}^{s, \tau}\left(\mathbf{R}^{n}\right)}\left\{\sum_{k \in \mathbf{Z}^{n}}(1+|k|)^{-N q / p}(d+|k|)^{n \tau q}\right\}^{1 / q} \lesssim\|f\|_{B_{P, q}^{s, \tau}\left(\mathbf{R}^{n}\right)},
\end{aligned}
$$

where we have chosen $N>n(\tau+1 / q) p$. Now, we turn to the case $q>p$. With $N>n(\tau p+1)$, we obtain

$$
\begin{aligned}
& \left\|\left\{t_{j, \ell}\right\}_{j, \ell \in \mathbf{Z}_{+}}\right\|_{b(p, q, \tau, \Omega)} \\
& \lesssim\left\{\sum_{k \in \mathbf{Z}^{n}}(1+|k|)^{-N} \sup _{P \in \mathcal{Q}} \frac{1}{|P|^{\tau p}}\left[\sum_{j=\max \left\{j_{P}, 0\right\}}^{\infty} 2^{j s q}\left[\left.\widetilde{\varphi}_{B\left(y_{P}, d \ell(P)+|k| \ell(P)\right)} * f(z)\right|^{p} d z\right]^{q / p}\right]^{p / q}\right\}^{1 / p} \\
& \lesssim\|f\|_{B_{p, q}^{s, \tau}\left(\mathbf{R}^{n}\right)}\left\{\sum_{k \in \mathbf{Z}^{n}}(1+|k|)^{-N}(d+|k|)^{n \tau p}\right\}^{1 / q} \lesssim\|f\|_{B_{p, q}^{s, \tau}\left(\mathbf{R}^{n}\right)} .
\end{aligned}
$$

Hence, we have proved that any $f \in B_{p, q}^{s, \tau}\left(\mathbf{R}^{n}\right)$ admits an atomic decompositions with respect to the sequence of coverings $\left\{\left\{\Omega_{j, \ell}\right\}_{\ell \in \mathbf{Z}_{+}}\right\}_{j \in \mathbf{Z}_{+}}$such that (5.3) holds true with some constants behind $\lesssim$ independent of $f$.

Substep 2.2. Now we study the regularity of appropriate atomic decompositions with coefficient sequence belonging to $b(p, q, \tau, \Omega)$. Associated to the sequence of coverings, $\left\{\left\{\Omega_{j, \ell}\right\}_{\ell \in \mathbf{Z}_{+}}\right\}_{j \in \mathbf{Z}_{+}}$, there exists a regular sequence of coverings by balls $\left\{\left\{B_{j, \ell}\right\}_{\ell \in \mathbf{Z}_{+}}\right\}_{j \in \mathbf{Z}_{+}}$such that

$$
\Omega_{j, \ell} \subset B_{j, \ell}, \quad j \in \mathbf{Z}_{+}, \quad \ell \in \mathbf{Z}_{+} ;
$$

see [33, Proposition 1]. Any atomic decomposition with respect to $\left\{\left\{\Omega_{j, \ell}\right\}_{\ell \in \mathbf{Z}_{+}}\right\}_{j \in \mathbf{Z}_{+}}$ represents trivially an atomic decomposition with respect to $\left\{\left\{B_{j, \ell}\right\}_{\ell \in \mathbf{Z}_{+}}\right\}_{j \in \mathbf{Z}_{+}}$. But, for such a standard covering, the desired property is known to be true, we refer to [53, Theorem 3.3]. Let us mention that this has been the only place, where we use $\tau \leq 1 / p$ in Step 2. This finishes the proof of Theorem 2.15.

\section{References}

[1] Alvarez, J., J. Lakey, and M. Guzmán-Partida: Spaces of bounded $\lambda$-central mean oscillation, Morrey spaces, and $\lambda$-central Carleson measures. - Collect. Math. 51, 2000, 1-47.

[2] Bergh, J., and J. Löfström: Interpolation spaces. An introduction. Springer, Berlin, 1976.

[3] Besov, O. V., V. P. IL'in, and S. M. Nikol'skiJ: Integral representations of functions and imbedding theorems. Volumes I and II. - V. H. Winston \& Sons, Washington, D.C., 1978, 1979.

[4] Besov, O. V., V. P. IL'in, and S. M. Nikol'skiJ: Integral'nye predstavleniya funktsii i teoremy vlozheniya. - Fizmatlit "Nauka", Second ed., Moscow, 1996 (in Russian).

[5] Burenkov, V.I., and E. D. Nursultanov: Description of interpolation spaces for local Morrey-type spaces. - Tr. Mat. Inst. Steklova 269, 2010, Teoriya Funktsii i Differentsialnye Uravneniya, 52-62; English transl. in Proc. Steklov Inst. Math. 269, 2010, 46-56.

[6] Chо, Y., and T. Ozawa: Sobolev inequalities with symmetry. - Commun. Contemp. Math. 11, 2009, 355-365. 
[7] Coleman, S., V. Glazer, and A. Martin: Action minima among solutions to a class of euclidean scalar field equations. - Comm. Math. Phys. 58, 1978, 211-221.

[8] Dafni, G., and J. XiaO: Some new tent spaces and duality theorems for fractional Carleson measures and $Q_{\alpha}\left(\mathbf{R}^{n}\right)$. - J. Funct. Anal. 208, 2004, 377-422.

[9] El BARAKA, A.: An embedding theorem for Campanato spaces. - Electron. J. Differential Equations 66, 2002, 1-17.

[10] El Baraka, A.: Function spaces of BMO and Campanato type. - In: Proc. of the 2002 Fez Conference on Partial Differential Equations, Electron. J. Differ. Equ. Conf. 9, 2002, 109-115.

[11] El BaraKa, A.: Littlewood-Paley characterization for Campanato spaces. - J. Funct. Spaces Appl. 4, 2006, 193-220.

[12] Epperson, J., and M. Frazier: An almost orthogonal radial wavelet expansion for radial distributions. - J. Fourier Anal. Appl. 1, 1995, 311-353.

[13] Epperson, J., and M. Frazier: Polar wavelets and associated Littlewood-Paley theory. Dissertationes Math. (Rozprawy Mat.) 348, 1996, 1-51.

[14] Essén, M., S. Jansson, L. PenG, and J. XiaO: $Q$ spaces of several real variables. - Indiana Univ. Math. J. 49, 2000, 575-615.

[15] Frazier, M., and B. Jawerth: Decomposition of Besov spaces. - Indiana Univ. Math. J. 34, $1985,777-799$.

[16] Frazier, M., and B. Jawerth: A discrete transform and decompositions of distribution spaces. - J. Funct. Anal. 93, 1990, 34-170.

[17] Haroske, D. D., and L. Skrzypczak: Continuous embeddings of Besov-Morrey function spaces. - Acta Math. Sin. (Engl. Ser.) 28, 2012, 1307-1328.

[18] Haroske, D. D., and L. Skrzypczak: Embeddings of Besov-Morrey spaces on bounded domains. - Studia Math. (to appear).

[19] Kozono, H., and M. Yamazaki: Semilinear heat equations and the Navier-Stokes equation with distributions in new function spaces as initial data. - Comm. Partial Differential Equations 19, 1994, 959-1014.

[20] KÜhn, T., H.-G. Leopold, W. Sickel, and L. SkrzypczaK: Entropy numbers of Sobolev embeddings of radial Besov spaces. - J. Approx. Theory 121, 2003, 244-268.

[21] Kuzin, I., and S. Pohozaev: Entire solutions of semilinear elliptic equations. - Birkhäuser Verlag, Basel, 1997.

[22] Liang, Y., Y. Sawano, T. Ullrich, D. Yang, and W. Yuan: New characterizations of Besov-Triebel-Lizorkin-Hausdorff spaces including coorbits and wavelets. - J. Fourier Anal. Appl. 18, 2012, 1067-1111.

[23] Liang, Y., D. Yang, W. Yuan, Y. Sawano, and T. UllRich: A new framework for generalized Besov-type and Triebel-Lizorkin-type spaces. - Dissertationes Math. (Rozprawy Mat.) 489, 2013, 1-114.

[24] Lions, P. L.: Symétrie et compacité dans les espaces de Sobolev. - J. Funct. Anal. 49, 1982, $315-334$.

[25] Mazzucato, A.: Besov-Morrey spaces: Function space theory and applications to non-linear PDE. - Trans. Amer. Math. Soc. 355, 2003, 1297-1369.

[26] Peetre, J.: New thoughts on Besov spaces. - Duke Univ. Press, Durham, 1976.

[27] Sawano, Y.: Wavelet characterization of Besov-Morrey and Triebel-Lizorkin-Morrey spaces. - Funct. Approx. Comment. Math. 38, 2008, 93-107.

[28] Sawano, Y., and H. Tanaka: Decompositions of Besov-Morrey spaces and Triebel-LizorkinMorrey spaces. - Math. Z. 257, 2007, 871-904.

[29] Sawano, Y., D. Yang, and W. Yuan: New applications of Besov-type and Triebel-Lizorkintype spaces. - J. Math. Anal. Appl. 363, 2010, 73-85.

[30] Sickel, W.: Radial subspaces of Besov-Lizorkin-Triebel spaces. - In: Proceedings Nonlinear Analysis, Function Spaces and Applications 9 (edited by J. Rakosnik), Inst. of Math., Academy of Scienes, Czech Rep., 2011, 169-215. 
[31] Sickel, W.: Smoothness spaces related to Morrey spaces - a survey. I. - Eurasian Math. J. 3, 2012, 110-149.

[32] Sickel, W.: Smoothness spaces related to Morrey spaces - a survey. II. - Eurasian Math. J. 4, 2013, 82-124.

[33] Sickel, W., and L. Skrzypczak: Radial subspaces of Besov and Lizorkin-Triebel classes: extended Strauss lemma and compactness of embeddings. - J. Fourier Anal. Appl. 6, 2000, $639-662$.

[34] Sickel, W., and L. Skrzypczak: On the interplay of regularity and decay in case of Radial functions II. Homogeneous spaces. - J. Fourier Anal. Appl. 18, 2012, 548-582.

[35] Sickel, W., and L. Skrzypczak: Subradial functions and compact embeddings. - Proc. Steklov Inst. Math. (to appear).

[36] Sickel, W., L. Skrzypczak, and J. Vybiral: On the interplay of regularity and decay in case of Radial functions I. Inhomogeneous spaces. - Commun. Contemp. Math. 14:1, 2012, 1250005, 1-60.

[37] SkrzypczAK, L.: Atomic decompositions on manifolds with bounded geometry. - Forum Math. 10, 1998, 19-38.

[38] Skrzypczak, L.: Rotation invariant subspaces of Besov and Triebel-Lizorkin space: compactness of embeddings, smoothness and decay of functions. - Rev. Mat. Iberoam. 18, 2002, 267-299.

[39] Strauss, W. A.: Existence of solitary waves in higher dimensions. - Comm. Math. Phys. 55, 1977, 149-162.

[40] TAng, L., and J. Xu: Some properties of Morrey type Besov-Triebel spaces. - Math. Nachr. 278, 2005, 904-914.

[41] Triebel, H.: Theory of function spaces. - Birkhäuser Verlag, Basel, 1983.

[42] Triebel, H.: Theory of function spaces II. - Birkhäuser Verlag, Basel, 1992.

[43] Triebel, H.: Theory of function spaces III. - Birkhäuser Verlag, Basel, 2006.

[44] TRIEBEL, H.: Local function spaces, heat and Navier-Stokes equations. - EMS Tracts Math. 20, Eur. Math. Soc., Zürich, 2013.

[45] Xiao, J.: Holomorphic $Q$ classes. - Lecture Notes in Math. 1767, Springer, Berlin, 2001.

[46] XiAo, J.: Geometric $Q_{p}$ functions. - Birkhäuser Verlag, Basel, 2006.

[47] YAnG, D., and W. YuAn: A new class of function spaces connecting Triebel-Lizorkin spaces and $Q$ spaces. - J. Funct. Anal. 255, 2008, 2760-2809.

[48] YAng, D., and W. YuAn: New Besov-type spaces and Triebel-Lizorkin-type spaces including $Q$ spaces. - Math. Z. 265, 2010, 451-480.

[49] YANG, D., and W. YuAN: Characterizations of Besov-type and Triebel-Lizorkin-type spaces via maximal functions and local means. - Nonlinear Anal. 73, 2010, 3805-3820.

[50] YAnG, D., and W. YuAn: Relations among Besov-type spaces, Triebel-Lizorkin-type spaces and generalized Carleson measure spaces. - Appl. Anal. 92, 2013, 549-561.

[51] YAnG, D., and W. YUAN: Function spaces of Besov-type and Triebel-Lizorkin-type-a survey. - Appl. Math. J. Chinese Univ. Ser. B (to appear).

[52] Yang, D., W. YuAn, and C. Zhuo: Complex interpolation on Besov-type and TriebelLizorkin-type spaces. - Anal. Appl. (Singap.) 11:5, 2013, 1350021, 1-45.

[53] Yuan, W., W. Sickel, and D. Yang: Morrey and Campanato meet Besov, Lizorkin and Triebel. - Lecture Notes in Math. 2005, Springer-Verlag, Berlin, 2010.

[54] Yuan, W., W. Sickel, and D. Yang: Compact embeddings for radial and subradial subspaces of some Besov-type spaces related to Morrey spaces. - J. Approx. Theory 174, 2013, 121-139. 